OPEN ACCESS

Edited by:

Sven Krappmann,

Friedrich-Alexander-Universität

Erlangen-Nürnberg, Germany

Reviewed by:

Wenbing Yin,

Institute of Microbiology (CAS), China

Ozgur Bayram,

Maynooth University, Ireland

*Correspondence:

Ingo Baver

ingo.bauer@i-med.ac.at

Stefan Graessle

stefan.graessle@i-med.ac.at

${ }^{t}$ These authors have contributed equally to this work

Specialty section:

This article was submitted to

Fungi and Their Interactions,

a section of the journal

Frontiers in Microbiology

Received: 23 May 2018

Accepted: 30 August 2018

Published: 19 September 2018

Citation:

Pidroni A, Faber B, Brosch G, Bauer I and Graessle S (2018) A Class 1

Histone Deacetylase as Major Regulator of Secondary Metabolite Production in Aspergillus nidulans.

Front. Microbiol. 9:2212.

doi: 10.3389/fmicb.2018.02212

\section{A Class 1 Histone Deacetylase as Major Regulator of Secondary Metabolite Production in Aspergillus nidulans}

\author{
Angelo Pidronit, Birgit Faber ${ }^{\dagger}$, Gerald Brosch, Ingo Bauer* and Stefan Graessle* \\ Division of Molecular Biology, Medical University of Innsbruck, Innsbruck, Austria
}

An outstanding feature of filamentous fungi is their ability to produce a wide variety of small bioactive molecules that contribute to their survival, fitness, and pathogenicity. The vast collection of these so-called secondary metabolites (SMs) includes molecules that play a role in virulence, protect fungi from environmental damage, act as toxins or antibiotics that harm host tissues, or hinder microbial competitors for food sources. Many of these compounds are used in medical treatment; however, biosynthetic genes for the production of these natural products are arranged in compact clusters that are commonly silent under growth conditions routinely used in laboratories. Consequently, a wide arsenal of yet unknown fungal metabolites is waiting to be discovered. Here, we describe the effects of deletion of hosA, one of four classical histone deacetylase (HDAC) genes in Aspergillus nidulans; we show that HosA acts as a major regulator of SMs in Aspergillus with converse regulatory effects depending on the metabolite gene cluster examined. Co-inhibition of all classical enzymes by the pan HDAC inhibitor trichostatin A and the analysis of HDAC double mutants indicate that HosA is able to override known regulatory effects of other HDACs such as the class 2 type enzyme HdaA. Chromatin immunoprecipitation analysis revealed a direct correlation between hosA deletion, the acetylation status of $\mathrm{H} 4$ and the regulation of SM cluster genes, whereas $\mathrm{H} 3$ hyperacetylation could not be detected in all the upregulated SM clusters examined. Our data suggest that HosA has inductive effects on SM production in addition to its classical role as a repressor via deacetylation of histones. Moreover, a genome wide transcriptome analysis revealed that in addition to SMs, expression of several other important protein categories such as enzymes of the carbohydrate metabolism or proteins involved in disease, virulence, and defense are significantly affected by the deletion of HosA.

\footnotetext{
Keywords: histone deacetylases, secondary metabolites, histone modifications and chromatin structure, transcription factors, filamentous fungi, Aspergillus, antifungals
}

\section{INTRODUCTION}

In addition to their indispensable ecological role in the recycling of organic material, filamentous fungi produce of a variety of commercially used compounds such as pigments, polysaccharides, vitamins, organic acids, enzymes, and even foodstuff such as miso, sake, shoyu, or Quorn (Newman and Cragg, 2012; Harvey et al., 2015). The most interesting fungal products, however, are small 
bioactive molecules that aid these organisms to adapt to adverse environmental conditions or to repel predators or microbes competing for food sources or habitats. Some of these molecules are dreaded mycotoxins that, if spoiled food is consumed, have a variety of detrimental effects on humans, ranging from allergic reactions and symptoms of poisoning to the triggering of cancer, if a low-dose exposure occurs over a longer time period (Pitt, 2000). Other SMs, however, are important as drugs against bacterial or fungal infections, hypertension, migraine, rejection of transplanted organs, heart disease, and as cholesterol-lowering substances (Fox and Howlett, 2008). Since only a minority of these molecules is produced under standard laboratory conditions, fungal species are representing a comprehensive source of a multitude of potential useful SMs that remain to be discovered. For instance, the genome of the mold Aspergillus nidulans holds putative genetic information for production of at least 30 still unknown secondary metabolites (Yaegashi et al., 2014) and thus, mining for novel beneficial substances in Aspergillus ssp. and many other fungal species is a major goal of mycologists worldwide. In the last decade, a huge number of strategies for the discovery of novel natural products of fungi have been developed. Among these strategies are optimized purification and screening techniques (e.g., Vansteelandt et al., 2013), a more sensitive detection of fungal metabolites (e.g., Klitgaard et al., 2015), and the application of microflow NMR coupled to untargeted mass spectrometry for structural identification of purified products (e.g., Bertrand et al., 2013). The fact that genes encoding enzymes involved in the biosynthesis of SMs such as polyketide synthases (PKSs), nonribosomal peptide synthetases (NRPSs), or terpene cyclases (TCs) are clustered on fungal chromosomes, does not only allow for their concerted regulation (Palmer and Keller, 2010) but also facilitates their detection from sequenced fungal genomes in silico, independently of the activity of certain SM clusters (e.g., van der Lee and Medema, 2016). Moreover, silent clusters can be induced by co-culturing of fungal strains together with another microorganism (e.g., Netzker et al., 2015) or via overexpression of specific transcription factors stimulating one or even more SM clusters. The latter approach led to the characterization of several novel metabolites in Aspergillus and several other species (e.g., Bergmann et al., 2007; Chiang et al., 2009; Chooi et al., 2013). An overview summarizing the multitude of emerging tools and technologies developed for the discovery of novel SMs was recently presented by Hautbergue et al. (2018) in a comprehensive review.

In recent years, however, also so-called "broad-domain regulators" such as LaeA (Bok et al., 2006), LaeB (Lin et al., 2018), or histone modifying enzymes (Shwab et al., 2007; Studt et al., 2016) were found to significantly impact the production of natural products in fungi. As in higher eukaryotes, genomic DNA of fungi is organized as chromatin and the extent of chromatin condensation determines the accessibility of genes or gene clusters for transcription factors. A complex combination of distinct histone modifications, termed as "histone code," contributes to structural changes of chromatin structure and thereby regulates the readout of the genetic information (Strahl and Allis, 2000). Among these modifications, acetylation of the
N-terminal core histone tails is of utmost importance. The dynamic process of acetylation involves two groups of enzymes: histone acetyl transferases (HATs) that transfer acetyl groups from acetyl-CoA to the $\varepsilon$-amino group of lysine residues and histone deacetylases (HDACs) that catalyze the removal of this modification and ensure the sensitive balance between acetylated and non-acetylated lysine residues of histones (Loidl, 1994; Verdin and Ott, 2015). Acetylation, however, not only acts as a specific signal on histones for the recruitment of distinct transcription factors (Loidl, 1994), but actually, those factors themselves may be substrates of HATs and HDACs (MartínezBalbás et al., 2000). The fact that several HDACs have also been identified in bacteria and archaea further indicates that these enzymes represent an evolutionary ancient protein family that access non-histone targets as well (Leipe and Landsman, 1997).

Aspergilli and other filamentous fungi exhibit genes encoding between two and four $\mathrm{NADP}^{+}$-dependent "sirtuin" type HDACs, summarized as class 3 enzymes (Brosch et al., 2008) and four so-called "classical HDACs" homologous to the class 1 enzymes RpdA and HosA (Graessle et al., 2000) and the class 2 enzymes HdaA and HosB, respectively (Trojer et al., 2003). Catalytic activity of the classical enzymes can be inhibited by a group of hydroxamate-containing molecules such as trichostatin A (TSA) and its FDA-approved derivatives belinostat, panobinostat, and vorinostat (Zhang et al., 2018). The significance of classical HDACs in gene regulation of higher eukaryotes has turned these inhibitors into a promising novel category of anti-cancer drugs with an increasing therapeutic potential also for the treatment of other diseases (Yoon and Eom, 2016). Since the class 1 enzyme RpdA was recently found to be essential for growth and sporulation of (pathogenic) filamentous fungi, HDAC inhibitors are currently also tested as antifungal substances for the treatment of invasive fungal infections in immunosuppressed patients (Tribus et al., 2010). Moreover, fungal HDACs and their inhibitors gained additional significance since only 2 years after its first characterization (Tribus et al., 2005), the class 2 enzyme HdaA was shown to be involved in the repression of certain SM clusters in A. nidulans (Shwab et al., 2007). This finding was confirmed for HdaA homologs in several other species (e.g., Lee et al., 2009) and led to the exploitation of HDAC inhibitors for the activation of cryptic SM clusters in Aspergillus, Alternaria, Penicillium, and Cladosporium (e.g., Shwab et al., 2007; ElHawary et al., 2018). However, as most of these "pan-inhibitors" are affecting all classical HDACs, it is difficult to attribute an effect to a specific enzyme. In addition, HDAC inhibitors might cause severe growth retardation in several fungal species, due to inhibition of the essential class 1 enzyme RpdA (Bauer et al., 2016) that probably causes indirect effects on SM production. In contrast, analyses of specific HDAC deletion mutants do allow insights into the functional role of single enzymes and, with suitable multiple deletion strains, even into possible interactions between different HDACs or HDAC classes.

We have previously demonstrated that deletion of the class 1 HDAC HDC1 of the maize pathogenic fungus Cochliobolus carbonum drastically reduced its virulence due to diminished expression of glucanase, polygalacturonase, and xylanase (Baidyaroy et al., 2001). Further analysis suggested that HDC1 
might be directly involved in the activation of these genes, a previously unexpected and novel role of a histone deacetylase.

Here, we show that HosA, a so far not studied class 1 HDAC of $A$. nidulans, functions as both, an activator and a repressor of SM production. Our studies revealed that depletion of catalytic HosA activity supports the production of several novel bioactive molecules; in contrast others, most notably the antibiotic penicillin, are strictly dependent on HosA activity. Hence, HosA has, in addition to its classical role as a repressor, an activating function as well. Moreover, RNASeq analysis revealed that regulation of several other gene categories is affected by HosA. Among those categories are genes involved in carbon metabolism, disease, virulence and defense, and detoxification processes. However, in contrast to its yeast homolog HOS2 (Pfaller et al., 2009), HosA has no impact on fungal resistance against azole derivatives or other conventional antifungal substances.

\section{MATERIALS AND METHODS}

\section{Fungal Strains and Growth Media}

Strains used in this study are listed in Supplementary Table S1. Strains were grown on glucose minimal medium (GMM) with appropriate supplerments as described (Cove, 1966). Xylanase promoter $(x y l P p)$ driven alleles were induced by addition of various concentrations $(0.1-1 \%)$ of xylose to GMM (GMMX).

\section{Generation of Fungal Strains}

Deletion of hos $A$ was performed as described previously (Tribus et al., 2005). The selection marker $\arg B$ was used for hos $A$ deletion in strain A89. Positive hosA deletion strains were confirmed by PCR screening and single integration of the hos $A$ deletion construct was verified by Southern analysis as described elsewhere (Graessle et al., 2000). For the complementation of $\operatorname{hos} A$, the hos $A$ delta strain TBF53.1 was co-transformed with a construct containing the hos $A$ encoding sequence including the endogenous promoter and a plasmid comprising a phleomycin selection marker bleR, respectively (Austin et al., 1990). Two independent transformants (TBFl2.3 and TBFz7.1) that showed integration of the complementation construct at differing genetic loci were identified by Southern analysis and expression of hos $A$ was verified via Northern analysis (data not shown).

Expression of His-, GFP-, and TAP-tagged (Bayram et al., 2012) HosA was achieved by an exchange of the endogenous hos $A$ regulatory sequence for the inducible/repressible heterologous xylanase promoter $(x y l P p)$ of Penicillium chrysogenum as described previously (Tribus et al., 2010). Expression of His-tagged HosA in the strain A89 was performed by targeted integration of the expression construct at the $\arg B$ locus as described in Lubertozzi and Keasling (2006). Three independent strains (TBF111, TBF117, and TBF122) carrying single integrations of the expression construct were identified by Southern and Northern blot analysis and expression of HosA was verified by immunoblot analysis using an anti-HosA antibody. For both, the GFP- and the TAP-tagged HosA expression, the hosA deletion strain TBF53.1 was used as recipient, and transformation was performed as co-transformation of the expression constructs together with a bleR carrying plasmid as described above. Expression of GFP- and TAP-tagged HosA was verified by immunoblot analysis using an anti-GFP and an antiCBP antibody, respectively. Two independent expression strains of each of HosA-TAP (TBFXT1.1 and TBFXT3.1) and HosAGFP (TBFGFP1.1 and TBFGFP4.1) were chosen for further analyses. For the expression of GFP-tagged RpdA, strain TIB54.1 was generated by targeted integration of pIB54 at the $\operatorname{argB}$ locus of strain A89 as described (Lubertozzi and Keasling, 2006).

\section{Sexual Crosses of Aspergillus nidulans}

Generation of hosA/hdaA double knock out strains was performed as described previously (Todd et al., 2007) by crossing of a hosA deletion strain (TBF53.1) with a hdaA deletion strain (H4). Double mutant strains RBF115 and RBF117 were identified on selective media and verified by PCR.

\section{Antifungal Susceptibility Testing (ETEST ${ }^{\circledR}$ )}

To compare drug susceptibility of hosA deletion and the wt strains ETEST ${ }^{\circledR}$ (bioMérieux) was employed. ETEST ${ }^{\circledR}$ consists of a predefined gradient of antifungal drugs on plastic strips. For this work ETEST ${ }^{\circledR}$ strips containing fluconazole (SKU number 412349), voriconazole (SKU number 412489), amphotericin B (SKU number 526348), and caspofungin (SKU number 412268) were used. Strains were grown on GMM at $37^{\circ} \mathrm{C}$ for $48 \mathrm{~h}$, spores were harvested and counted and a sterile swab was dipped into the spore suspension adjusted to $10^{6}$ conidia per $\mathrm{ml}$. The inoculum was plated onto solid GMM and left to dry for $15 \mathrm{~min}$ at RT before the application of Etest strips ${ }^{\circledR}$. Drug susceptibility of strains was evaluated after $24 \mathrm{~h}$ and $48 \mathrm{~h}$ of growth at $37^{\circ} \mathrm{C}$.

\section{Northern and Western Analyses}

Expression analyses of tagged HosA versions were performed under $x y l P \mathrm{p}$ inductive ( $1 \%$ glucose/1\% xylose) and repressive ( $1 \%$ glucose) conditions in minimal medium. RNA preparation, blotting, and hybridization were done as described (Graessle et al., 2000). Dig-dUTP-labeled DNA probes specific for the corresponding transcripts to be quantified were amplified with primers shown in Supplementary Table S2. Hybridized probes were detected by Anti-Digoxigenin-AP Fab fragments (Roche) and developed with CSPD chemiluminescent substrate (Roche) according to the manufacturer's instructions. Signals were visualized by exposure to X-ray film or with the Fusion-SL 3500 WL imaging system (Vilber Lourmat). Total protein extracts were prepared by grinding 50-100 mg of lyophilized mycelia with a Tungsten Carbide ball in a mixer mill (Retsch ${ }^{\circledR}, \mathrm{MM}$ 400) followed by extraction with 250-500 $\mu$ l of buffer B250 as described in Bayram et al. (2012). Western blotting and detection was performed as described (Trojer et al., 2003). Proteins were detected by antibodies directed against HosA (Trojer et al., 2003) or anti-CBP (Millipore 07-482, 1:1333).

\section{Bioassay of Penicillin Activity}

Bioassays were performed as described in Bok and Keller (2004) to determine penicillin (PN) activity. Fungal strains were grown 
in liquid medium, with an inoculum density of $5 \times 10^{6}$ conidia per milliliter. After removal of the mycelium by filtration, the medium was lyophilized and resuspended in 1/5 volume of sterile A. dest. The PN sensitive Kocuria rhizophila strain ATCC9341 was grown in $\mathrm{BD}^{\mathrm{TM}} \mathrm{BBL}^{\mathrm{TM}}$ Trypticase ${ }^{\mathrm{TM}}$ Soy Broth until the culture reached an $\mathrm{OD}_{600}$ of 1.0 . For the assay plates, $50 \mathrm{ml}$ precooled $\left(46^{\circ} \mathrm{C}\right)$ antibiotic medium 1 ( Roth $\left.^{\circledR}\right)$ was mixed with $3.75 \mathrm{ml}$ of $K$. rhizophila culture and poured into $14.5 \mathrm{~cm}$ petri dishes. Wells ( $\varnothing 8 \mathrm{~mm}$ ) were pierced into the test plates and $100 \mu \mathrm{l}$ of each sample were applied to wells. Control samples were pretreated with $15 \mathrm{U}$ of penicillinase from Bacillus cereus (SigmaAldrich ${ }^{\circledR}$ ). Assay plates were incubated at $4^{\circ} \mathrm{C}$ for $2 \mathrm{~h}$, to allow initial diffusion of the samples and subsequently incubated over night at $37^{\circ} \mathrm{C}$.

\section{Purification of HosA Activity and HDAC Assay}

For purification of HosA-TAP, six $1000 \mathrm{ml}$ Erlenmeyer flasks containing $200 \mathrm{ml}$ each of GXMM media were inoculated with A. nidulans conidia $\left(5 \times 10^{6} / \mathrm{ml}\right)$ and incubated with shaking at $37^{\circ} \mathrm{C}$ for $15 \mathrm{~h}$. Affinity purification until the first elution by TEV protease was performed as described (Bayram et al., 2012). Aliquots of the elution were directly used for HDAC assays or frozen in liquid nitrogen for storage at $-80^{\circ} \mathrm{C}$.

Enzymatic activity of enriched HosA was measured in triplicates using either $\left[{ }^{3} \mathrm{H}\right]$ acetate-prelabeled chicken histones as substrate (Trojer et al., 2003) or fluorometric labeled peptides of a commercial (EMD Millipore) HDAC Assay Kit according to the manufacturer's instructions. Briefly, $15 \mu$ l of the IgG eluate were mixed with HDAC Assay Buffer containing either Trichostatin A [TSA] in several concentrations (5, 20, 100, 250, 500 , and $750 \mathrm{nM}$ ), or DMSO as control and subsequently was incubated for $60 \mathrm{~min}$ at $25^{\circ} \mathrm{C}$. After addition of $20 \mu \mathrm{l}$ activator solution, samples were further incubated for $15 \mathrm{~min}$ at $20^{\circ} \mathrm{C}$. Fluorescence was measured using a FLUOstar Omega Plate Reader (BMG Labtech) set to 355 and $460 \mathrm{~nm}$ for excitation and emission, respectively. Before use, the instrument was tested and calibrated by creating a standard curve as described in the protocol of the HDAC assay kit.

\section{Subcellular Localization of HosA}

To determine the subcellular localization of HosA, the HosAGFP expressing strain, was grown at $30^{\circ} \mathrm{C}$ overnight, on cover slips in six-well plates, under conditions of moderate $(0.1 \%$ xylose) induction of the $x y l P$ promoter. As the cellular localization of RpdA was previously shown to be predominantly nuclear (Bauer et al., 2016), a strain expressing RpdA-GFP under the control of $x y l P p$ (TIB54.1) was used as reference. DNA was stained with DAPI.

\section{RNA-Seq Analysis}

Strains were grown in triplicates in $100 \mathrm{ml}$ of GMM at a density of $2 \times 10^{6} / \mathrm{ml}$ at $37^{\circ} \mathrm{C}$ for 24 and $60 \mathrm{~h}$, respectively. cDNA libraries of 12 samples were sequenced on the Illumina platform (Illumina HiSeq Single Read sequencing) in which reads of $50-b p$ were generated. The resulting reads were aligned using TopHat (version 2.1.1) (Trapnell et al., 2012) to the A. nidulans FGSC_A4 genome (version s10-m04-r06) received from the AspGD database. Gene expression levels were calculated with the Cufflinks package (version 2.2.1) and normalized by the number of fragments per kilobase of exon per million mapped reads (FPKM). Differential gene expression analysis was performed with Cuffdiff (version 2.2.1) (Trapnell et al., 2012). Tables containing expression values of each gene received from the Cuffdiff analysis were filtered $(\log 2 \mathrm{FC} \geq 2$, FDR < 0.05) and prepared for MIPS functional catalog (FunCat) (Ruepp et al., 2004), ontology enrichment analysis by using the FungiFun2 platform (Priebe et al., 2015). FunCat categories with a false discovery rate (FDR) under 0.05 were defined as significantly enriched. The FDR correction of each directly and indirectly annotated top category was calculated using the Benjamini-Hochberg method. Data processing and generation of graphical plots were performed using $\mathrm{R}$ (version 3.5.0) with the packages dplyr (Wickham et al., 2017), ggplot2 (Wickham, 2009), pheatmap (Kolde, 2015), and VennDiagram (Chen, 2018). In general, schemes of SM gene clusters were generated using the illustrator for biological sequences (Liu et al., 2015).

\section{ChIP Analysis and qPCR}

Chromatin immunoprecipitation analysis was performed as described (Boedi et al., 2012) with minor modifications. GMM medium was inoculated with $A$. nidulans conidia $\left(10^{6} / \mathrm{ml}\right)$ and incubated at $30^{\circ} \mathrm{C}$ for $48 \mathrm{~h}$. Crosslinking of proteins and DNA was induced by the addition of formaldehyde to a final concentration of $0.8 \%(\mathrm{w} / \mathrm{v})$ and stopped with $750 \mathrm{mM}$ TRIS-HCl [pH 8] after $15 \mathrm{~min}$ of further shaking. Chromatin of extracts of $300 \mathrm{mg}$ of lyophilized mycelia was sheared in $1.5 \mathrm{ml}$-TPX tubes (Diagenode) using the Bioruptor plus (Diagenode) in cycles for $30 \mathrm{~s}$ "on" and $30 \mathrm{~s}$ "off" at maximal power. To produce soluble chromatin with average size between 200 and $600 \mathrm{bp}$, samples were sonicated between 5 and 10 cycles. After sonication tubes were centrifuged for $1 \mathrm{~min}$ at $15.000 \times g$ at $4^{\circ} \mathrm{C}$, supernatants were used in further steps. After pre-clearing, $300 \mu \mathrm{g}$ of protein were incubated with $3 \mu \mathrm{g}$ of antibody over night at $4^{\circ} \mathrm{C}$ on a rotary shaker. The protein-antibody conjugate was precipitated with $30 \mu \mathrm{l}$ of paramagnetic Protein A beads (Dynabeads, Invitrogen) for $1 \mathrm{~h}$ at $4^{\circ} \mathrm{C}$ on a rotary shaker. Unspecific proteins were removed by two subsequent washes with $1 \mathrm{ml}$ of washing buffer $(0.2 \%$ SDS, 0.5\% Triton X-100, 2 mM EDTA, 20 mM Tris- $\mathrm{HCl} \mathrm{pH} 8$ ) containing $150 \mathrm{mM} \mathrm{NaCl}$ and $500 \mathrm{mM} \mathrm{NaCl}$, respectively. The chromatin-Dynabeads complex was washed twice in $1 \mathrm{ml}$ of TEbuffer (1 mM EDTA, $10 \mathrm{mM}$ Tris- $\mathrm{HCl} \mathrm{pH}$ 8). For resuspension of the chromatin, $50 \mu \mathrm{l}$ of fresh resuspension buffer (1\% SDS, $0.1 \mathrm{M} \mathrm{NaHCO}_{3}$ ) was added, incubated at $65^{\circ} \mathrm{C}$ for $15 \mathrm{~min}$, and centrifuged for $2 \mathrm{~min}$. After repeating this step, supernatants were collected in a final volume of $100 \mu \mathrm{l}$. For reverse crosslinking, samples were incubated with $4 \mu \mathrm{l}$ of $\mathrm{NaCl}(5 \mathrm{M})$ overnight at $65^{\circ} \mathrm{C}$. After this step, $2 \mu \mathrm{l}$ EDTA $(0.5 \mathrm{M}), 4 \mu \mathrm{l}$ TRIS-HCl $(1 \mathrm{M}$, $\mathrm{pH} 6.5)$, and $2 \mu \mathrm{l}$ proteinase $\mathrm{K}$ was added and incubated for $1 \mathrm{~h}$ at $45^{\circ} \mathrm{C}$. To remove RNA, samples were treated with RNase A (3\% in total) and incubated at $65^{\circ} \mathrm{C}$ for $5 \mathrm{~min}$ and at $37^{\circ} \mathrm{C}$ for $30 \mathrm{~min}$. DNA was purified with a PCR purification kit (QIAGEN) and eluted in $80 \mu \mathrm{l}$ TE-buffer. For qPCR, DNA was used undiluted 
and in a 1:5 dilution whereas input controls were used in a 1:100 dilution.

Real-time PCR was performed with an ABI Prism 7900HT Detection System (Applied Biosystems). Standard curves were generated using eluted DNA from ChIP-experiments in serial dilutions. Two microliter of template was used in a 20- $\mu \mathrm{l}$ total volume reaction using Thermo Fisher Power SYBR ${ }^{\mathrm{TM}}$ Green PCR master mix. PCR was performed in triplicates for each single ChIP experiment using primer pairs specific for three 5-prime regions of each of $\operatorname{ors} A, \operatorname{ipn} A, \operatorname{atn} A$, and $c i c B$ (see Supplementary Table S2). To calculate the signal of enrichment for each region, percent of input normalization was used $\left(100 \times 2^{\wedge}(\right.$ Adj.input $\left.-C t[I P])\right)$, whereas Adj.input $=C t$ (input) $-\log 2$ (dilution factor); average and standard deviation were generated from these values.

\section{RESULTS}

\section{HosA Deletion Causes Morphological Effects and Production of Pigments but Does Not Affect Resistance Against Antifungal Substances}

In yeast and certain molds it was shown recently that the class 1 HDAC HOS2 contributes to resistance against antifungals such as voriconazole (Pfaller et al., 2009). This observation is of enormous medical interest, since efficacy of azoles used in antifungal therapies could be increased by combination with HDAC inhibitors, substances that recently gained importance as anti-cancer drugs (Dokmanovic et al., 2007). In order to investigate the role of HOS2-type enzymes in Aspergilli, the coding sequence of the HOS2 orthologous enzyme HosA was deleted. Spores of independent hosA deletion strains with a single genomic integration of the deletion construct were plated onto agar plates and ETEST strips (bioMérieux) loaded with increasing concentrations of the antifungal substances voriconazole, fluconazole, amphotericin B, and caspofungin, respectively, were applied to the plates. Deletion strains complemented for hos $A$ were used as wildtype controls in the sensibility testing.

Interestingly, examination of the ETEST plates after $48 \mathrm{~h}$ at $37^{\circ} \mathrm{C}$ revealed that hos $A$ in $A$. nidulans did not lead to the expected increase of sensitivity against any of the antifungals tested. Indeed, sensitivity of the corresponding mutants against fluconazole seemed to be marginally decreased in some cases (Supplementary Figure S1). When spores were dotted onto plates without antifungal substances, however, growth and sporulation of colonies of the mutants were diminished and mycelia showed a significant reddish-brown coloration on the bottom side of the colonies (Figure 1A). Interestingly, a similar coloration was observed in the shaking culture supernatant of hos $A$ delta strains grown for $24 \mathrm{~h}$ at $37^{\circ} \mathrm{C}$ (Figure 1B). Moreover, microscopic inspections revealed a significant hyper-branching of hyphae of the HosA mutants (Figure 1A). These results demonstrate that deletion of HosA does not alter the sensibility of

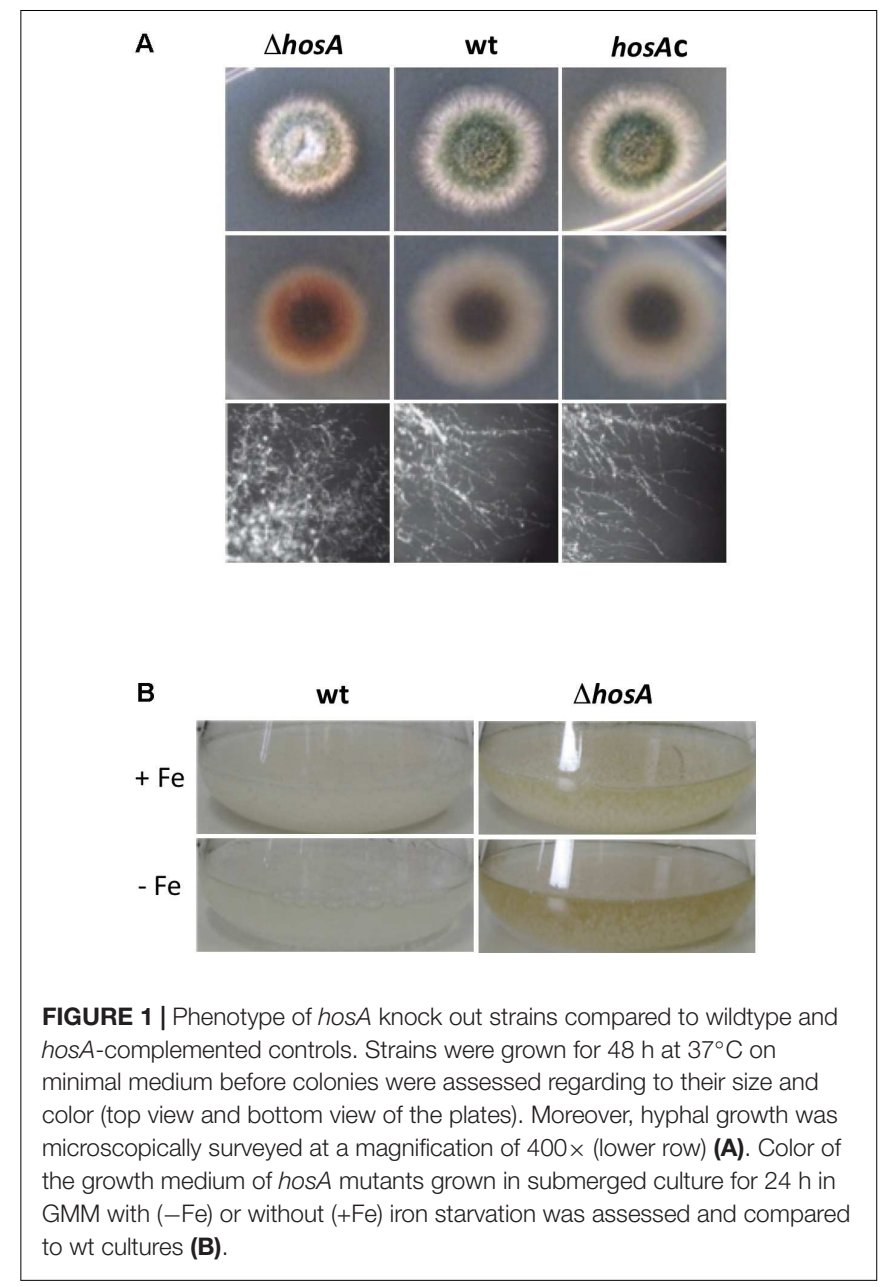

A. nidulans to selected antifungal drugs but leads to a diminished growth and discoloration of hyphae and media.

\section{HosA Acts as Repressor of the Orsellinic Acid Gene Cluster}

The striking pigmentation of HosA deletion strains on agar plates and in submerged culture suggested a possible role of HosA as repressor of secondary metabolites, as many of them are molecules contributing to the pigmentation of mycelia or are excreted to the culture medium. Two of such metabolites, the cathepsin K inhibitors F-9775A and F-9775B were identified recently as yellow-orange pigments of $A$. nidulans. Both compounds, however, are not produced under standard culture conditions but are induced in co-culture with the bacterial species Streptomyces hygroscopicus (Schroeckh et al., 2009) or under nitrate and orthophosphate limitation (Sarkar et al., 2012). Structural analyses of these two polyketides suggest that they are derivatives of the fungal archetypal polyketide orsellinic acid (OA). In order to prove a possible impact of HosA in the regulation of the $\mathrm{OA}$ biosynthetic gene cluster, hosA deletion strains were grown for 24, 36, 48, and $60 \mathrm{~h}$ and RNA was used for Northern blot analyses with a hybridization probe for ors $A$, a gene coding for a PKS of the OA biosynthetic gene cluster. 


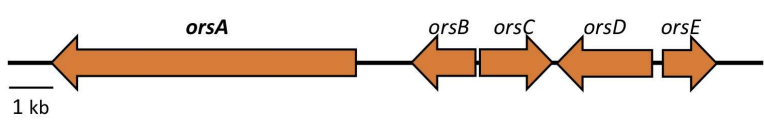

A

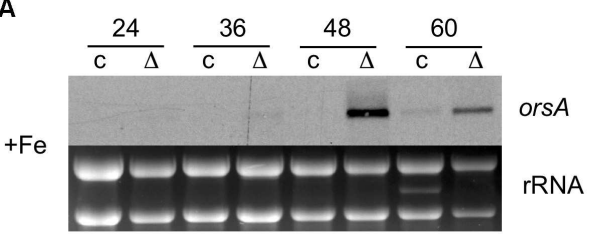

B

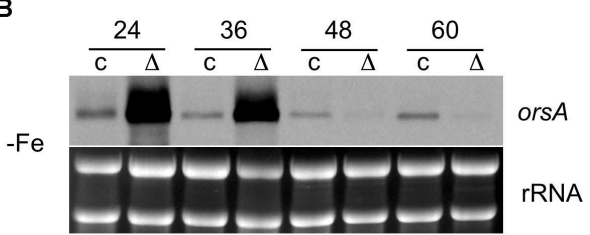

C

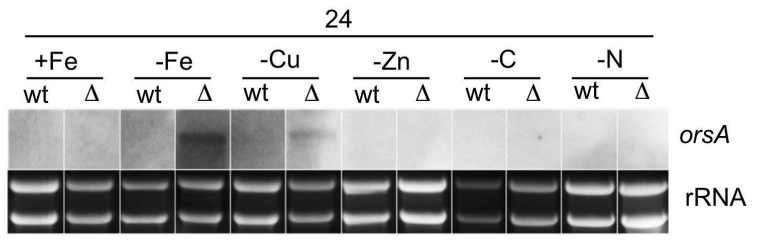

FIGURE 2 | Expression of orsellinic acid biosynthetic cluster genes in hosA knock out strains and wildtype after different times of growth and under different starvation conditions. A schematic representation of the five cluster genes (ors $A-E$ ) is shown on the top of the figure, an ors $A$ probe (bold) was used for Northern analysis. For ors $A$ expression analysis, $\triangle$ hos A mutants and complemented strains were grown for $24,36,48$ and $60 \mathrm{~h}$ at $37^{\circ} \mathrm{C}$ in a submerged culture (GMM) without (A) or with (B) iron starvation. Thirty micrograms of total RNA were botted, ethidium-stained rRNA was used as quality and loading control. orsA expression was also analyzed with $10 \mu \mathrm{g}$ of RNA of hosA mutants and wt grown for $24 \mathrm{~h}$ under iron $(-\mathrm{Fe})$, copper $(-\mathrm{Cu})$, or zinc $(-\mathrm{ZN})$ starvation and under carbon- $(-\mathrm{C}, 0.1 \%$ glucose) or nitrogen- (-N, 2 mM Gln) limitation (C).

After $36 \mathrm{~h}$ of growth, hosA-deleted strains showed weak and after $48 \mathrm{~h}$ strong transcription of ors $A$, whereas no transcription was detectable in the complemented control strains (Figure 2A). After $60 \mathrm{~h}$ of growth, however, a very weak transcript could be detected in the complemented strains as well, suggesting that not only deletion of hosA but also depletion of nutrients during growth might influence the regulation of the $\mathrm{OA}$ gene cluster. In order to prove this assumption, a HosA mutant and a wildtype strain were grown for $24 \mathrm{~h}$ under different starvation conditions. No effects were detected under zinc starvation or under carbon or nitrogen limitation (Figure 2C). Under copper or iron starvation, however, ors $A$ transcription was moderately or considerably induced already in early hos $A$ delta cultures and even in hosA-complemented strains and the wildtype, a weak ors $A$ transcript was detected when $30 \mu \mathrm{g}$ instead of $10 \mu \mathrm{g}$ of RNA were loaded (Figure 2B and data not shown). These results demonstrate that transcription of ors $A$ is induced under hos $A$ depleted conditions and is further enhanced under copper and iron starvation.

\section{Trichostatin A Effectively Inhibits Catalytic HosA Activity and Induces Expression of the Orsellinic Acid Biosynthetic Gene Cluster}

Since OA expression was found to be stimulated in co-culture with Streptomyces strains (Schroeckh et al., 2009), bacteria known as producers of the HDAC inhibitor trichostatin A (TSA), we wondered, if OA biosynthetic cluster genes can be induced by TSA via the inhibition of HosA activity. In order to analyze the sensitivity of catalytic HosA activity to TSA, the enzyme was TAP-tagged as described (Bayram et al., 2012), expressed under the control of the heterologous xylanase promoter $x y l P \mathrm{p}$ of $P$. chrysogenum in strain TBFXT1.1 (see Supplementary Table S1) and pulled down with IgG-Sepharose under native conditions. Eluted fractions were then analyzed by immunoblotting using an anti-CBP antibody as described (Bauer et al., 2016). Aliquots of enriched HosA were subsequently assayed for HDAC activity with increasing concentrations of TSA. Catalytic HosA activity was reduced by more than $60 \%$ when TSA was added to $100 \mathrm{nM}$ final concentration. At $500 \mathrm{nM}$ TSA, more than $95 \%$ of HosA activity was inhibited demonstrating its sensitivity to this inhibitor (Figure 3A).

This result prompted us to check the in vivo effect of TSA with regard to the transcription of the three OA cluster genes $\operatorname{ors} A, \operatorname{ors} C$, and ors $E$, respectively. To this end, an A. nidulans wild type was grown for $21 \mathrm{~h}$ in a submerged culture before $1 \mu \mathrm{M}$ of TSA was added to the medium for $3 \mathrm{~h}$. An untreated wildtype and a hos $A$ delta strain were used as controls. RNA was prepared and analyzed with probes against the three OA cluster genes in a Northern blot. As shown in Figure 3B, treatment with TSA leads to an upregulation of the transcripts in the wildtype, very similar to that found in the hosA delta strain without TSA. No induction of the corresponding cluster genes was detectable in the wildtype control with DMSO. These results confirm a direct correlation between HosA activity and expression of OA biosynthetic cluster genes. 


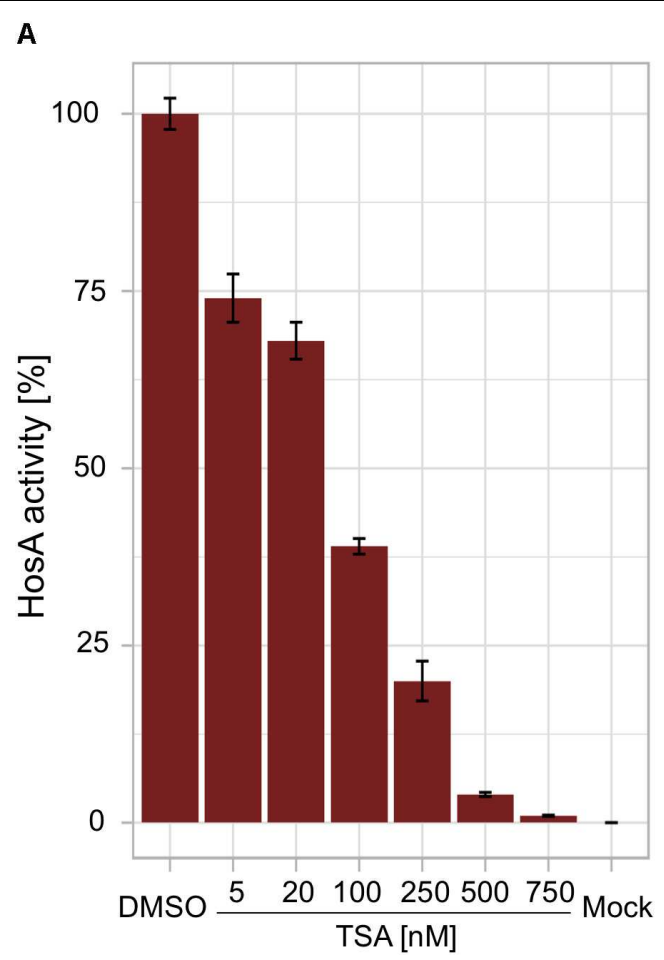

B

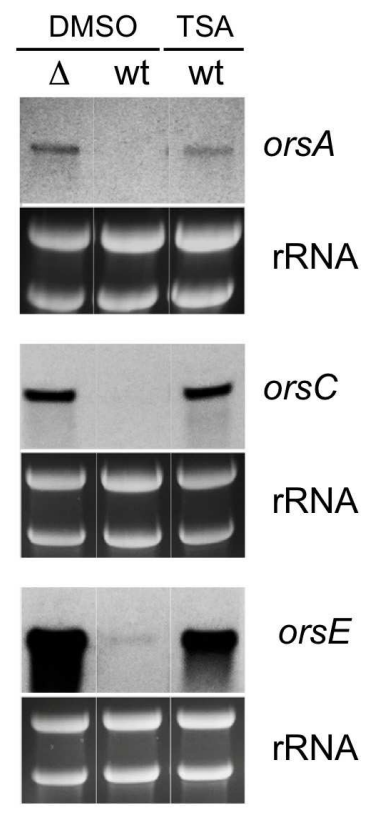

FIGURE 3 | Effect of trichostatin A on the catalytic activity of HosA and the expression of orsellinic acid cluster genes. HDAC activity of purified TAP-tagged HosA was eluted by cleavage with TEV protease and aliquots were used for HDAC activity assays with increasing concentrations of the HDAC inhibitor trichostatin A (TSA). HosA activity with dimethyl sulfoxide (DMSO), the solvent of TSA, was set to 100\%, an assay aliquot without recombinant HosA was used as mock control (A). Expression of the orsellinic acid cluster genes ors $A$, ors $C$, and ors $E$ in a wildtype strain with and without TSA treatment. hosA mutant and wt strains were grown in a submerged culture (GMM) for $21 \mathrm{~h}$ at $37^{\circ} \mathrm{C}$ under iron starvation before $1 \mu \mathrm{M}$ TSA (or the corresponding amount of DMSO for the controls) was added to the cultures for additional $3 \mathrm{~h}$. After harvesting of the strains, total RNA was prepared from the mycelia, blotted onto a Hybond-N membrane and hybridized with a digoxigenin-labeled probe against ors A, orsC, and orsE, respectively (B).

\section{Production of Penicillin Is HosA-Dependent}

The fact that HosA activity represses OA biosynthesis prompted us to ask for the role of HosA for the production of other important SMs, such as penicillin $(\mathrm{PN})$. In order to analyze if $\mathrm{PN}$ production is induced beyond wildtype level in hos $A$ deletion mutants, a bacterial growth inhibition assay was performed as described (Shwab et al., 2007). Since we have shown that the class 2 type enzyme $\mathrm{HdaA}$ acts as repressor of $\mathrm{PN}$ production in Aspergillus (Shwab et al., 2007), we also were interested, if the induced PN production of the $h d a A$ delta strain can be further increased by an additional deletion of hos $A$. To address both questions, we generated $h d a A / h o s A$ double knock out strains and analyzed them, together with the $h d a A$ single knock out, in the bacterial growth inhibition assay. Compared to the wildtype control, the hdaA delta strain showed the expected increase in $\mathrm{PN}$ production after 48 and $60 \mathrm{~h}$ (Figure 4A). Unexpectedly, however, $\mathrm{PN}$ concentration in the growth medium of HosA mutants was not increased but decreased below the detection level of the bioassay. This rather surprising finding was also confirmed by results of the hdaA/hosA double mutants suggesting that the elimination of $\mathrm{PN}$ production by hos $A$ deletion overrides PN upregulation caused by the deletion of $h d a A$. In order to verify these findings on the transcriptional level, Northern analysis was performed. The strain deleted for hos A, a hos A-complemented strain, an hdaA/hosA double mutant, and a wildtype control were grown for 48 and $60 \mathrm{~h}$ before RNA was prepared and hybridized with a probe against ipnA, encoding for isopenicillin $\mathrm{N}$ synthase. In contrast to the wildtype and the hos $A$ complemented strain that showed a clear ipnA transcript, no ipnA transcript was detectable in hos $A$ and in $h d a A / h o s A$ mutants after 48 and $60 \mathrm{~h}$ (Figure 4B), confirming the findings of the bacterial growth inhibition assay. Hence, HosA is absolutely required for sufficient expression of ipnA in A. nidulans, even in the absence of HdaA.

The fact that a recent study revealed that class 1 HDAC complexes in yeast can promote nucleosome assembly independently of activity (Chen et al., 2012) raised the question, if HosA per se or its catalytic activity is required for the production of PN. To address this question, Northern analyses under TSA treatment were performed. A wildtype strain was grown for 43 or $41 \mathrm{~h}$ in submerged culture before $1 \mu \mathrm{M}$ TSA was added to the medium for 5 or $7 \mathrm{~h}$. A culture treated solely with the TSA solvent DMSO was used as negative control. After $5 \mathrm{~h}$ of TSA treatment, ipnA transcription was significantly reduced compared to the control and after $7 \mathrm{~h}$, transcription was beyond 


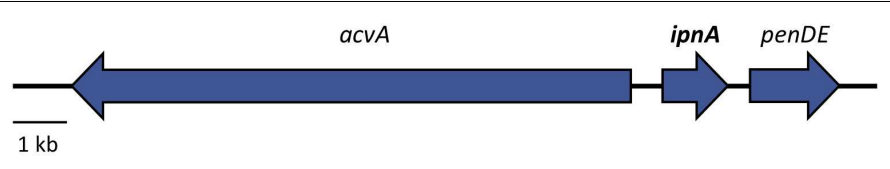

A

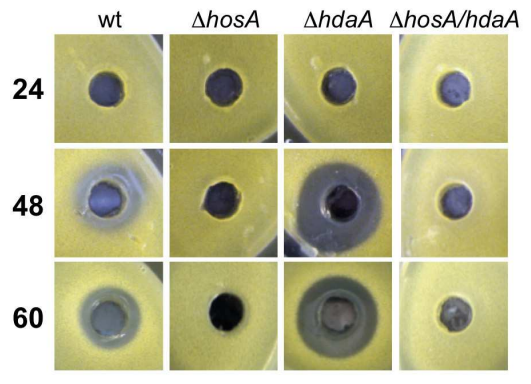

B

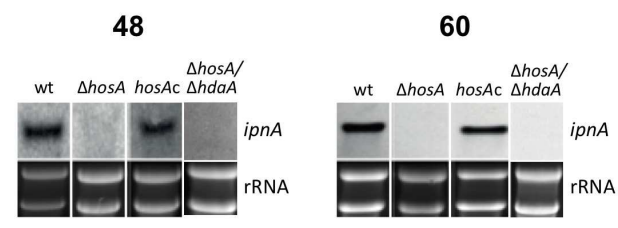

C

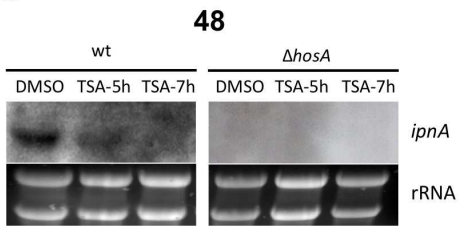

FIGURE 4 | Production of penicillin and expression of the penicillin cluster gene ipnA in different HDAC mutants. A bacterial growth inhibition assay was used for quantification of penicillin production in $h o s A-, h d a A-$, and hosA/hdaA-delta strains compared to wildtype. Strains were grown for 24,48 , and $60 \mathrm{~h}$ at $37^{\circ} \mathrm{C}$ in a submerged culture in GMM before media (sixfold concentrated) were pipetted into wells of agar plates inoculated with the PN sensitive bacteria strain $K$. rhizophila (ATCC9341). After incubation over night at $37^{\circ} \mathrm{C}$, the size of the bacterial growth inhibition zone was analyzed. Bacterial clearing around the well corresponds to the relative production of penicillin of the strains tested (A). Northern blots were used to verify the results of the bioassay. hos $A$ and hos $A / h d a A$ mutants, a complemented hosA mutant and wt were grown for 48 and $60 \mathrm{~h}$ at $37^{\circ} \mathrm{C}$ in submerged GMM cultures. RNA of strains was hybridized with a digoxigenin-labeled probe for the penicillin cluster gene ipnA (see schematic representation of the gene cluster on top of this figure). Ethidium-stained rRNA served as quality and loading control (B). Impact of TSA on the penicillin production was assessed. hosA mutant and the wt strain grown for 43 and $41 \mathrm{~h}$ in GMM were treated with $1 \mu \mathrm{M}$ TSA for 5 and 7 h. RNA was prepared, blotted, and hybridized with the ipnA probe. DMSO treated cultures served as negative controls (C).

the detection level (Figure 4C). As expected, no transcript was detectable in a hosA-deleted control strain.

\section{HosA Overexpression Does Not Affect Penicillin Production but Leads to Minor Growth Retardation of the Expression Strains}

Since HosA activity is obviously required for sufficient ipnA expression, we were interested whether HosA overexpression leads to PN levels beyond wildtype. In order to address this question, strains TBF117 and TBF122, expressing His-tagged HosA under $x y l P p$ (see Supplementary Table S1), were grown in submerged culture supplemented with $1 \%$ xylose or $1 \%$ glucose to induce or repress HosA expression. Crude protein extracts of strains were analyzed by immunoblotting using an antiHosA antibody. In order to identify possible cross-reactions of the antibody with proteins of the crude extracts, a wildtype strain was used as negative control. Under repressive conditions, no signals were observed in the corresponding mutant strains, whereas a strong signal was detected under $x y l P p$ inductive conditions confirming an efficient and strong expression of recombinant HosA (Supplementary Figure S2A). As expected, no signal was detectable in crude protein extracts of the control strains due to the low expression levels of endogenous HosA (Trojer et al., 2003). RNA was prepared from the induced cultures and used for Northern analysis with a hybridization probe for $i p n A$ as described above. Interestingly, transcription level of ipnA did not differ from that of a wildtype control suggesting that PN production is not increased over wildtype level under HosA overexpression (data not shown). However, on agar plates, overexpression of HosA led to a minor but significant retardation of colony growth when compared to wildtype and hosA-complemented strains (Supplementary Figure S2B).

\section{HosA Is a Major Regulator of Secondary Metabolites in Aspergillus nidulans}

The significant but contrary results of hos A deletion on the production of $\mathrm{OA}$ and $\mathrm{PN}$ encouraged us to examine the effect of HosA on the transcriptome of $A$. nidulans with a specific focus on SM gene clusters. To this end, RNA from hos A delta mutants and complemented strains grown in liquid media for 24 and $60 \mathrm{~h}$ at $37^{\circ} \mathrm{C}$ was reversely transcribed, and cDNA was used for sequencing on an Illumina HiSeq platform. Three biological replicates were analyzed resulting in 12 RNA-Seq samples, a total of 714,529,862 high-quality short-sequence reads (approximately $50 \mathrm{bp}$ ) yielding $108 \mathrm{gb}$ of transcriptomic sequence data. Almost 650 million short reads $(90.9 \%)$ were successfully mapped to 
A

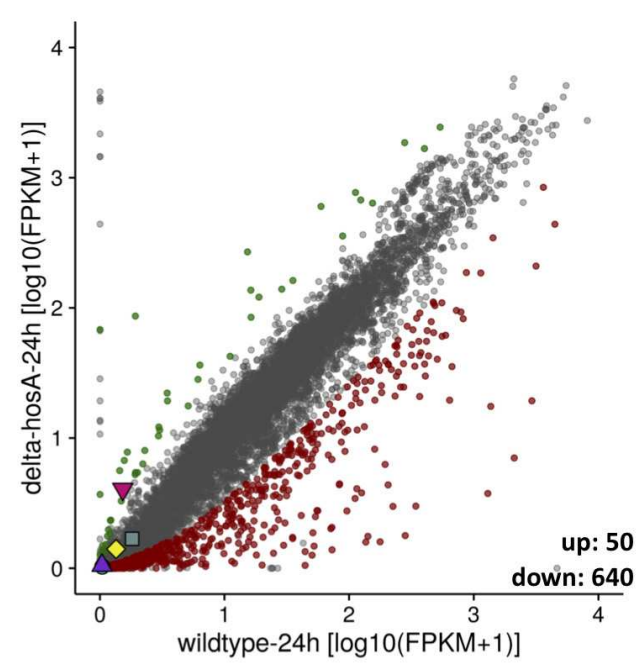

B

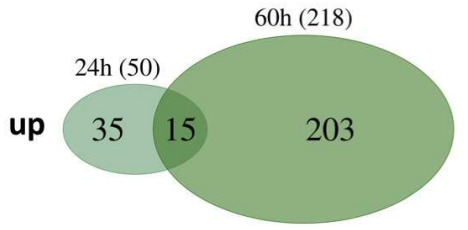

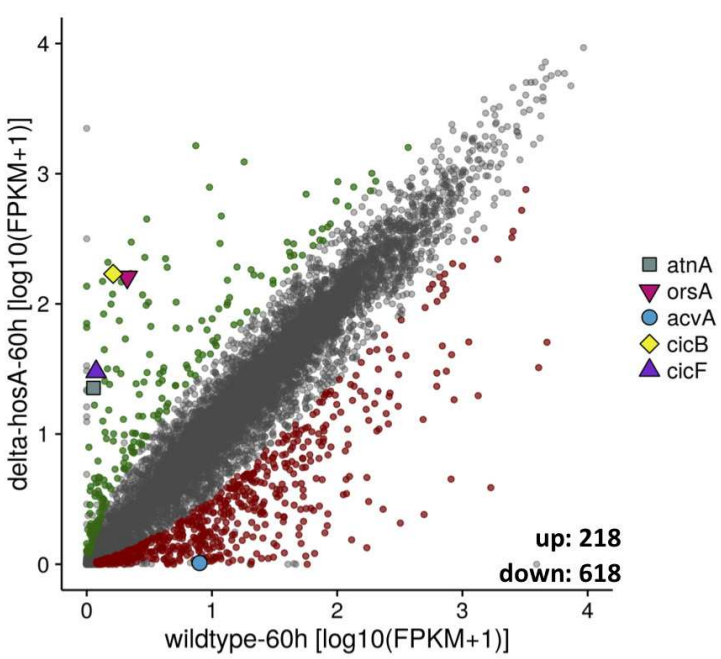

$\log 2 \mathrm{FC} \geq 2, \mathrm{FDR}<0.05, \mathrm{n}=10820$

FIGURE 5 | RNA-Seq analysis of differentially expressed genes in hosA mutants. Scatter plots show the expression values (log10[FPKM + 1]) for every annotated gene $(n=10.820)$ after 24 and $60 \mathrm{~h}$ of growth in $\mathrm{GMM}$ at $37^{\circ} \mathrm{C}$. An additional cutoff (log2FC $\left.>2 / F D R<0.05\right)$ for differentially expressed genes was applied. Dots within these cutoff criteria are displayed in gray and not considered as being affected by hos $A$ deletion. Green dots indicate significantly upregulated, red dots significantly downregulated genes in hosA mutants. Biosynthetic cluster members of orsellinic acid (orsA), aspercryptin (atnA), cichorine (cicB, cicF), and penicillin $(\operatorname{acvA})$ are highlighted, total number of up- and down-regulated genes are given (A). Venn diagrams below illustrate the distribution of up- (green) and down-regulated (red) genes in the hos A mutants in 24 or $60 \mathrm{~h}$ cultures. Numbers of overlapping and non-overlapping up- and downregulated genes are show (B).

the A. nidulans reference genome (Supplementary Table S3). Raw sequencing data and processed files are accessible via gene expression omnibus (Barrett et al., 2013) provided by the NCBI (accession number GSE117388).

Alignments were analyzed with Cufflinks (Trapnell et al., 2012) to conduct a genome-wide analysis of differential gene expression among hos $A$ deletion strains and a wildtype control. Cuffdiff analysis revealed that 4,839 and 7,221 out of 10,820 annotated genes show a significantly different expression after 24 and $60 \mathrm{~h}$ of growth, respectively. To reduce this huge number of affected genes to the most significant ones, an additional cutoff $(\log 2 \mathrm{FC} \geq 2, \mathrm{FDR}<0.05)$ was applied resulting in 50 and 640 high-significantly up- and down-regulated genes in the hos $A$ mutant after $24 \mathrm{~h}$ of growth. After $60 \mathrm{~h}, 218$ and 618 genes were significantly up- and down-regulated (Figure 5A and Supplementary Table S4). Interestingly, only a minority of 15 up- and 151 down-regulated genes were overlapping in the shortand the long-term culture (Figure 5B).
Among the down-regulated genes, also several SM cluster members showed diminished expression under hos $A$ deletion. One of them, acvA, encodes the delta-(L-alpha-aminoadipyl)-Lcysteinyl-D-valine synthetase of the PN cluster, confirming the results of our bacterial growth assays and the Northern analysis (Figure 4). Other genes associated with categories related to secondary metabolism, however, were significantly upregulated (Figure 5A). In addition to the already identified ors $A$ of the OA cluster (Figure 2), $c i c B$ and $c i c F$ of the cichorine (CC) cluster (Sanchez et al., 2012) and cluster members of novel secondary metabolites like aspercryptin (AC) (Chiang et al., 2016) were significantly upregulated by the deletion of hos $A$. To further confirm these results and to determine, if iron limitation leads to a similar enhancement of the HosA effect already shown for OA cluster genes, additional Northern experiments were performed with probes for $\operatorname{cic} B$ and $\operatorname{atn} G$, two representatives of the CC and the AC cluster, respectively. Under iron sufficient conditions, hosA deletion strains showed a strong upregulation 
A
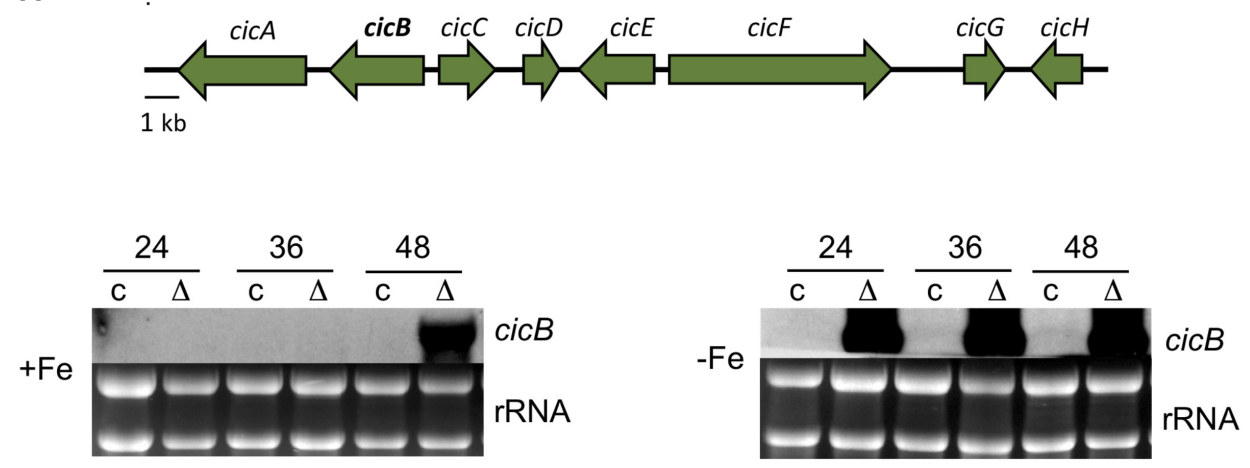

B
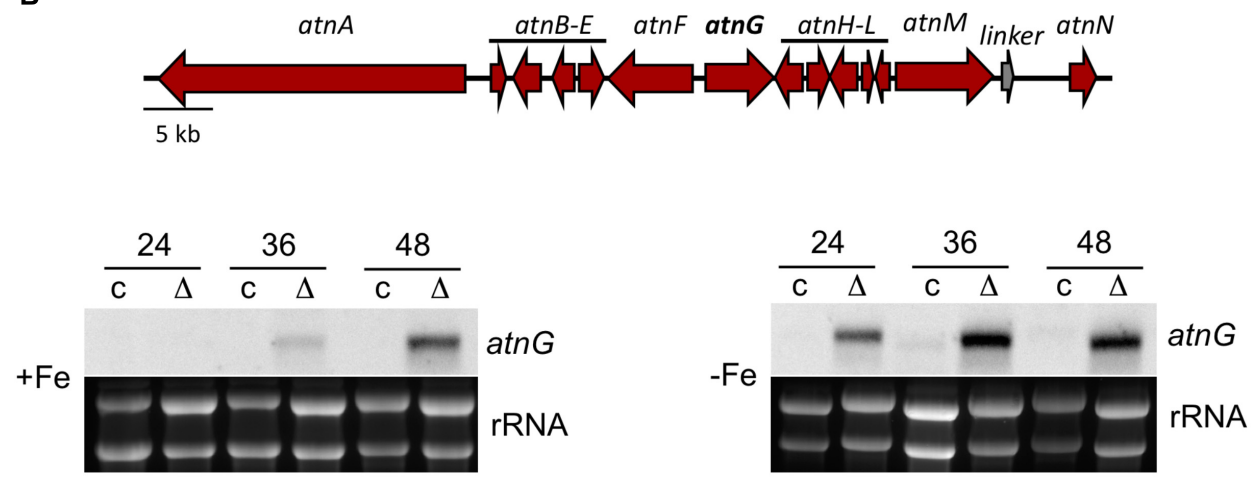

FIGURE 6 | Expression analysis of members of the cichorine and aspercryptin biosynthetic gene cluster in hos $A$ knock out mutants compared to hos $A$-complemented strains. A schematic representation of cluster genes of the cichorine- (cicA-H) and the aspercryptin- (atnA- $N$ ) gene cluster is shown on the top of the figure. For expression analysis, hosA mutants and complemented strains were grown for 24,36 , and $48 \mathrm{~h}$ at $37^{\circ} \mathrm{C}$ in $\mathrm{GMM}$ with (-Fe) or without (+Fe) iron starvation. Blotted RNA was hybridized with digoxigenin-labeled probes for the cichorine cluster gene cicB (A) and the aspercryptin cluster gene atn $\mathbf{G}$ (B). Ethidium-stained rRNA was used as quality and loading control.

of both genes after $48 \mathrm{~h}$, whereas no signals were detectable in the complemented controls (Figure 6). Under iron starvation, transcription of both, $c i c B$ and $\operatorname{atn} G$, was already induced during an earlier stage of fungal growth, however, was exclusively observed in hosA delta strains, confirming the RNA-Seq results and the impact of iron limitation on the production of SMs.

The significant role of HosA in the transcription of these SM cluster members prompted us to investigate, whether genes adjacent to the corresponding clusters are as well affected by the deletion of hosA. To address this question, RNA-Seq data of the four most prominently regulated SM clusters, OA, AC, $\mathrm{CC}$, and $\mathrm{PN}$, and five adjacent genes flanking both sides of each cluster were analyzed with regard to their transcription (Supplementary Table S6). Heatmaps of the cluster regions deduced from this analysis clearly illustrate that the regulatory effect of hos $A$ deletion is almost exclusively restricted to these clusters (Figure 7). Adjacent genes were, if at all, only barely affected indicating a very specific HosA driven regulation.

In order to get an idea about the general impact of HosA on the regulation of SMs in Aspergillus, the expression values of 70 confirmed or putative coding sequences of proteins responsible for the biosynthesis of secondary metabolites (Khaldi et al., 2010; Nielsen et al., 2011; Andersen et al., 2013) were evaluated based on our transcriptome data (Supplementary Table S5). A heatmap deduced from this list clearly illustrates that a considerable number of these so called "key synthases" displays significant differences in expression level in wildtype and hos A deletion strains after 24 and/or 60 h (Supplementary Figure S3). Among these enzymes are members of well-known SM clusters such as StcJ, a putative PKS of the sterigmatocystin (ST) cluster (Brown et al., 1996; Bok et al., 2006), XptA, a prenyltransferase required for the prenyl xanthone synthesis (Sarkar et al., 2012), MicA, an NRPS involved in the production of microperfuranone (Yeh et al., 2012), and SidD, an NRPS involved in siderophore-mediated iron uptake (Schrettl et al., 2008). In order to determine the significance of HosA in the regulation of whole SM clusters, the expression of 20 adjacent genes up- and downstream of each key synthase was evaluated based on our transcriptome data. It turned out that expression of some clusters like the sterigmatocystin cluster was not collectively changed, 

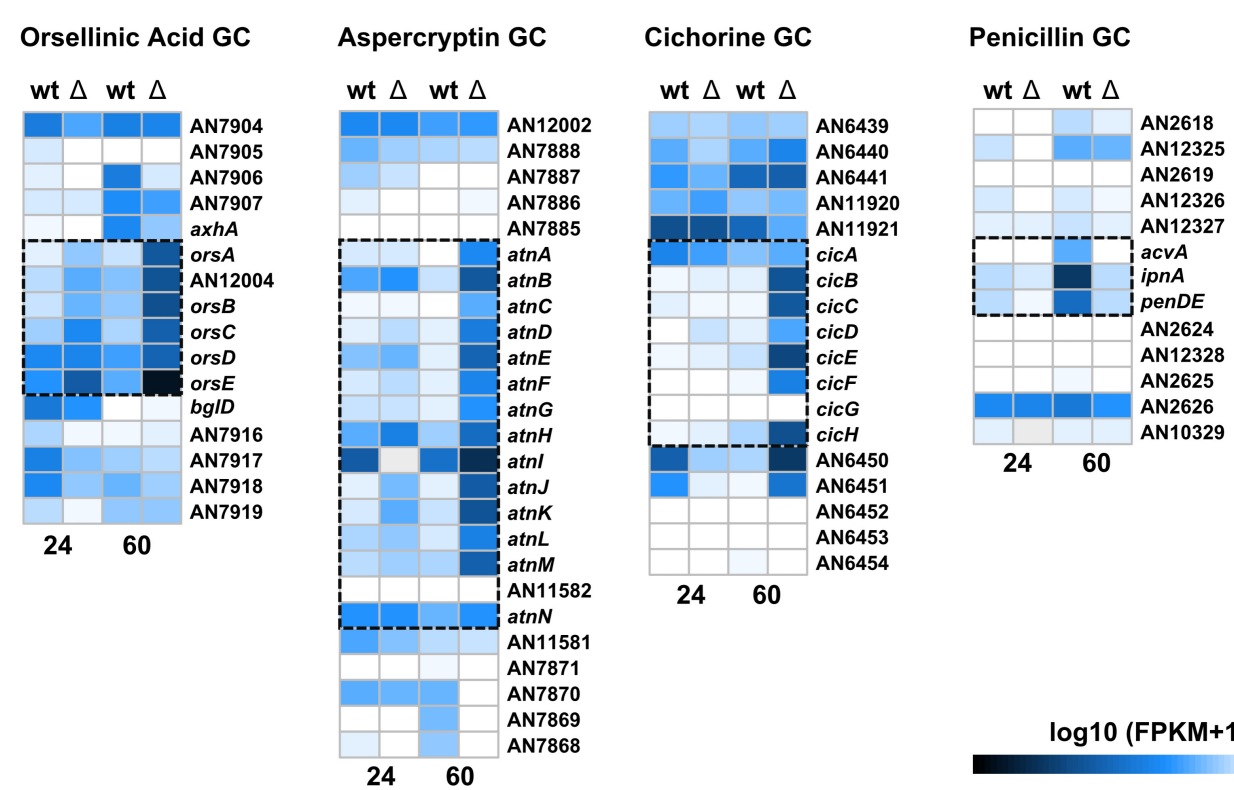

$\log 10(F P K M+1)$

$\begin{array}{lllllll}3 & 2.5 & 2 & 1.5 & 1 & 0.5 & 0\end{array}$

FIGURE 7 | Expression analysis of the most affected biosynthetic clusters in hosA deletion mutants. Heatmaps display expression of the orsellinic acid, aspercryptin, cichorine, and penicillin secondary metabolite gene cluster members and adjacent genes after 24 and $60 \mathrm{~h}$ in hosA mutants versus wildtype strains. The predicted genes of each cluster are boxed, FPKM +1 values are log10-transformed.

as would have been suggested by StcJ. Other clusters, however, displayed significant regulation, although the corresponding key synthase was not affected by the deletion of hosA. Finally, expression values of 25 out of 70 predicted NRPSs and PKSs (listed in Supplementary Table S5) and their adjacent genes were selected for presentation. As shown in Supplementary Table S7, HosA affects in addition to the OA, AC, CC, and the PN cluster the transcription of several additional clusters such as the emericellamide (easA/easB) cluster (Chiang et al., 2008), the aspyridone (apdA) cluster (Bergmann et al., 2007), the asperfuranone ( $a f_{o} E / a f_{o} G$ ) cluster (Chiang et al., 2009; Bergmann et al., 2010), and also cryptic clusters such as $p k h A / p k h B$ and inpA/inpB (Ahuja et al., 2012; Inglis et al., 2013).

In summary, our RNA-Seq data revealed HosA as a major regulator of SMs of $A$. nidulans with converse regulatory effects depending on the metabolite gene cluster examined. This is substantiated by the finding that at least 14 already characterized SM gene clusters show significant differential regulation in the hosA mutants. Iron deficiency further increases the regulatory effects of hos $A$ deletion and leads to a significantly earlier induction of the corresponding SM cluster genes.

\section{Chromatin Immunoprecipitation Analysis Suggests an Ambivalent Role of HosA in the Regulation of Fungal SMs}

In order to confirm an effect of HosA on the acetylation status of SM cluster regions, chromatin immunoprecipitation (ChIP) analysis was established. Since acetylated (ac) H3K9 is closely correlated with active promoters (Karmodiya et al., 2012) and H4K16ac promotes chromatin compaction and transcriptional repression in yeasts (Kurdistani and Grunstein, 2003; Wirén et al., 2005; Abshiru et al., 2016), an anti-H3K9ac- and an antiH4K16ac-antibody were chosen for the ChIP experiments. hosA-deleted mutants and hosA-complemented strains were grown for $48 \mathrm{~h}$ before formaldehyde was added to the shaking culture for crosslinking. After sonication, immunoprecipitation, reversed crosslinking, and protein and RNA digestion, purified DNA was used for quantitative PCR. To this end, three different 5-prime target regions (T1, T2, and T3, see Supplementary Table S2) of each of the cluster genes ors A (OA cluster), ipnA (PN cluster), cicB (CC cluster), and atnA (AC cluster), were considered for quantification. Interestingly, all cluster genes (including the repressed ipnA) showed significant hyper-acetylation of H4K16 under hosA deletion in all the regions analyzed (Figure 8B), while H3K9 hyper-acetylation was only detectable in ors $A$ and $\operatorname{atn} A$ in all three regions. Only one region (T1) of ipnA showed increased acetylation of H3K9 (Figure 8A), whereas no significant acetylation beyond wildtype level was observed for $c i c B$. Indeed, one region of $c i c B$, T1, actually showed significant hypo-acetylation on $\mathrm{H} 3 \mathrm{~K} 9$ in hosA mutants.

Taken together, our results indicate an important role of HosA in H4K16 deacetylation linked to repression of prominent SM clusters such as OA, CC, and AC. The fact that ipnA, although repressed in hosA deletion strains, is as well hyper-acetylated at H4K16 suggests that HosA acts in addition as a dominating inductor of some gene clusters - 




B

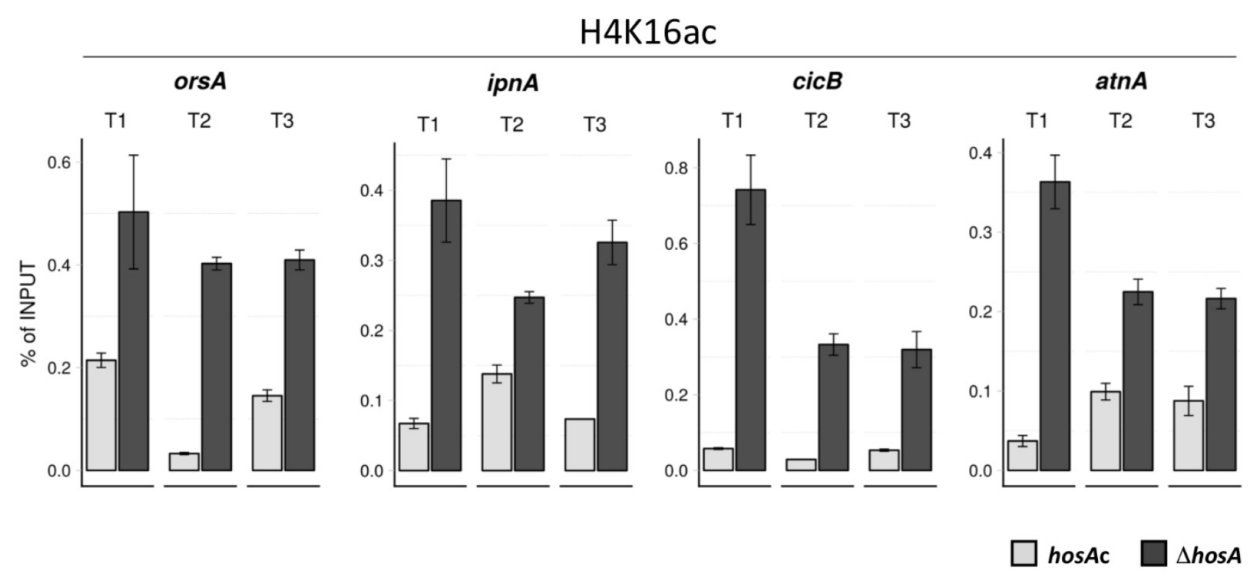

FIGURE 8 | ChIP analysis of H3K9 and H4K16 acetylation in hosA mutants and hosA-complemented strains. Bars indicate enrichment of complemented strains (light) and hosA mutants (dark) in percentage of the input-DNA used for ChIP analysis. The crosslinked protein-DNA-complex was incubated with antibodies against $\mathrm{H} 3 \mathrm{~K} 9 \mathrm{ac}(\mathbf{A})$ and H4K16ac (B), respectively. For each of the genes ors $A$, ipnA, cicB, and atnA, qPCR was performed using primer-sets specific for three targets ( $\mathrm{T} 1$, T2, T3) within the $5^{\prime}$ regions of the corresponding coding sequences. Error bars represent standard deviation calculated from triplets of each sample.

an uncommon function that remains to be studied in more detail. In contrast, as shown for $\operatorname{cicB}, \mathrm{H} 3$ hyper-acetylation is no absolute requirement for transcriptional activation of all gene clusters.

\section{DISCUSSION}

Filamentous fungi comprise several human pathogens that can cause serious infections such as invasive aspergillosis due to Aspergillus ssp., which may be severe and often life threatening in immunosuppressed patients (Lamoth et al., 2015). As key players in eukaryotic gene expression, HDACs are involved in the regulation of many proteins of fungi. Some of those represent virulence factors or essential molecules for fungal survival (Brosch et al., 2008). Inhibitors of HDACs, which are already exploited as potential therapeutic and chemo-preventive agents against cancer, neurodegenerative disorders, and graft intolerance, are also discussed as potential antifungal agents for the treatment of invasive fungal infections (Elaut et al.,
2007). Due to limited efficiency of classical antifungal drugs and an increasing resistance against established azole derivatives, there is an urgent need for alternative therapy regimes. A few years ago, a novel antifungal substance, MGCD290 (MethylGene Inc., Montreal, QC, Canada), was identified as a specific HOS2 inhibitor in Candida ssp. (Pfaller et al., 2009). As an antifungal agent, MGCD290 alone displayed only moderate activity, however, in combination with azoles, it significantly increased the in vitro susceptibility of fungal species such as Aspergillus fumigatus, Aspergillus flavus, Aspergillus niger, and Aspergillus terreus (Lamoth et al., 2015).

These results prompted us to delete hos $A$, the gene for the HOS2 homologue of $A$. nidulans, a so far not studied class 1 HDAC in Aspergilli. Subsequently, HosA deficient mutant strains were analyzed with regard to their sensitivity against azoles and other established antifungal substances. In contrast to the increased sensitivity observed under MGCD290 treatment (Pfaller et al., 2009), hosA deletion did not affect the efficacy of any antifungal tested. These contradictory results may be explained 
by either a different biological function of HosA type proteins in different Aspergillus strains and species or, more likely, by the fact that MGCD290 is not exclusively acting on HosA type enzymes in filamentous fungi but influences the function of other classical HDACs as well. One of these HDACs, RpdA, was recently found to be required for viability of the opportunistic pathogen A. fumigatus (Bauer et al., 2016) and therefore, diminished RpdA activity due to MGCD290 treatment might potentiate the antifungal effect of azoles. Similar synergies between azoles and inhibition of HDACs were observed in some Candida species (Smith and Edlind, 2002). In these strains, TSA treatment led to a reduced up-regulation of multi drug transporter- (CDR) and azole target-(ERG) genes; it was speculated that TSA treatment is associated with histone acetylation of the promoter region of repressors of the CDR- and ERG-genes. The responsible HDAC, however, remained undiscovered. Our results suggest that in A. nidulans, HosA has neither direct nor indirect effect on drug susceptibility to azoles and thus cannot be regarded as a preferential target for inhibitors in order to enhance efficacy of conventional antifungal therapies.

However, HosA deficiency or its catalytic inhibition by TSA did show a remarkable effect on the transcription of various SM clusters and, most likely, on the production of the corresponding secondary metabolites of A. nidulans. In general, regulation of SM clusters in filamentous fungi is complex and involves multiple protein complexes (Brakhage, 2013). Many transcription factors (TFs) are thereby located on the corresponding gene cluster itself and are specifically regulated by various environmental stimuli. In addition, also trans-acting TFs exist that crosstalk between different clusters even on different chromosomes (Bergmann et al., 2010). In recent years, it turned out that important regulators such as LaeA as well as histone modifying enzymes are also able to regulate the production of several SMs (Bok and Keller, 2004; Gacek and Strauss, 2012). The class 2 HDAC HdaA of $A$. nidulans was the first example of such a histone modifying enzyme. In $h d a A$ deletion strains, we could show a significantly increased production mainly of two secondary metabolites of $A$. nidulans: the carcinogenic aflatoxin precursor sterigmatocystin and the antibiotic penicillin (Shwab et al., 2007). Since addition of TSA to growth media of other fungal species such as Alternaria and Penicillium increased the production of several cryptic SMs as well, it was speculated that this upregulation is mainly due to the inhibition of the $\mathrm{HdaA}$ orthologues in these fungi (Shwab et al., 2007). The fact that HosA now has been proven as another major regulator of SMs in Aspergillus suggests, that the observed effects of HDAC inhibitors are most likely not exclusively due to HdaA inhibition. Indeed, our analysis of hosA/hdaA double mutants actually implies that the inductive effect on penicillin production via HdaA inhibition can even be overruled by the repressive effect of depleted HosA activity.

The finding that depletion (or inhibition) of an HDAC is silencing gene transcription is uncommon but not entirely unexpected. The first HDAC gene we have deleted in a filamentous fungus was $H D C 1$ in the plant pathogen C. carbonum (Baidyaroy et al., 2001). HDC1 was also the first example for a significant downregulation of specific genes (encoding extracellular depolymerases) in an HDAC mutant, resulting in non-pathogenic Cochliobolus mutants. Subsequently, upregulation of specific genes via HOS2 type proteins was substantiated in Saccharomyces cerevisiae (Wang et al., 2002) and recent investigations in the phytopathogenic fungus Fusarium fujikuroi also confirmed a significant negative effect on both, pathogenicity and production of four SMs in strains with deleted ffhda2, a hosA homolog of this fungus (Studt et al., 2013). In these mutants, production of three secondary metabolites, gibberellin, bikaverin, and fusarin, was decreased between 65 and $80 \%$ and expression of fusaric acid was almost completely abolished resembling the situation of penicillin in Aspergillus hosA delta strains. A similar unexpected effect was also observed recently by Fan et al. (2017), where deletion of an HAT in the entomopathogenic fungus Metarhizium robertsii led to the characterization of not less than 11 new fungal metabolites confirming an inductive effect of hypo-acetylation on the production of certain SM clusters.

In addition to its cryptic role as inducer of the penicillin cluster and several other genes associated to SM production or other protein categories (Figure 9 and Supplementary Table S8), HosA as well functions as a repressor of SMs in Aspergillus. Although the regulatory principle of HosA remains to be studied in detail for each gene cluster affected, several possibilities are conceivable of how such opposing effects on transcription could be achieved by one and the same enzyme. HosA might directly deacetylate specific lysines on histones $\mathrm{H} 3$ and $\mathrm{H} 4$, repressing transcription of the respective gene-region, as shown for instance for orsellinic acid and aspercryptin cluster genes (Figure 6). Indeed, it has been demonstrated for the orsellinic acid cluster that $\mathrm{H} 3 \mathrm{~K} 9$ acetylation, mediated by the Saga/Ada complex, might trigger its activation (Nützmann et al., 2011) and a recent publication reports on two HATs of the plant pathogenic fungus Fusarium graminearum required for the regulation of secondary metabolism via acetylation of several lysine residues of H3 (Kong et al., 2018).

On the other hand, there is evidence from yeast that HOS2, in addition to its classical role as a repressor, might directly induce transcription by the deacetylation of (hyper-)acetylated H3 and H4 sites (Wang et al., 2002), a conceivable possibility why penicillin cluster genes such as $i p n A$ are downregulated in hosA mutants. Alternatively, HosA might modify penicillinspecific TFs that induce genes independently of the acetylation level of the corresponding histones. It is known that probably all HDACs are able to target non-histone proteins such as transcriptional regulators as well (Glozak et al., 2005). The fact that catalytic activity of purified HosA is rather low when measured in vitro with acetylated chicken histones as substrate [(Trojer et al., 2003) and data not shown], but is significantly increased when acetylated peptides were used (Figure 3), strengthens the assumption that histones are not the predominate substrates of HosA. In addition, GFP-tagged HosA was detectable in both, the nucleus and the cytoplasm of the hyphae, also indicating additional non-histone targets of HosA (Supplementary Figure S4). This observation is in 

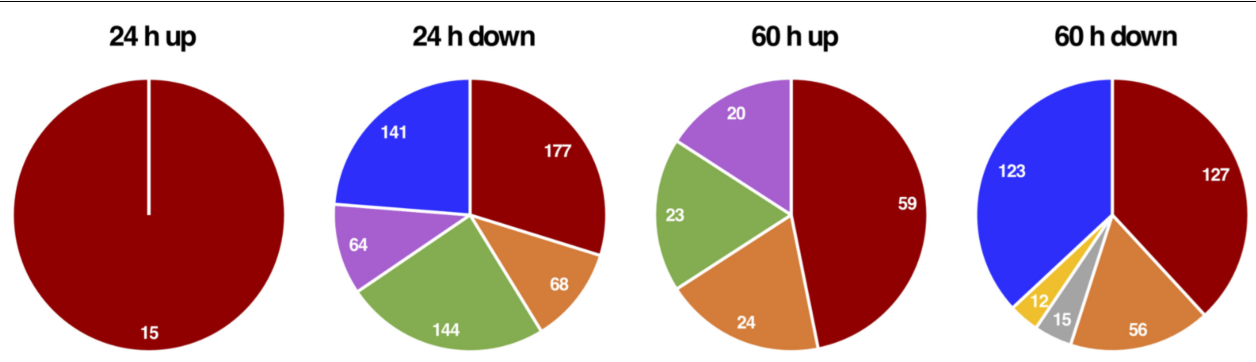

- secondary metabolism - =transported compounds (substrates) - Gcompound and carbohydrate metabolism = disease, virulenœ and defense - detoxification = extracellular metabolism = degradation / modification of foreign (exogenous) compounds

FIGURE 9 | Most significantly enriched FunCat gene categories of hosA mutant strains versus wildtype. Differentially expressed genes in the RNA-Seq analysis were used for a MIPS Functional Catalogue (FunCat) ontology analysis on the FungiFun2 platform (also see Supplementary Table S8). Pie charts illustrate the most significantly enriched FunCat categories of down- and up-regulated genes after 24 and 60 h of growth from hierarchy level 2 . Numbers of genes related to the significant categories are displayed within the pie charts.

accordance with results from yeast, where HOS2 was shown to shuttle between the cytoplasm and the nucleus via a chaperone dependent process (Liu et al., 2012). Moreover, during the preparation of this manuscript, a paper was published that presented clear evidence that a HosA ortholog in the insectpathogenic fungus Beauveria bassiana is indirectly involved in both, global acetylation and phosphorylation of H3K56 and $\mathrm{H} 2 \mathrm{AS} 129$, respectively, providing further evidence of an indirect effect of HosA-type proteins in the regulation of fungal transcription via deacetylation of HATs or kinases (Cai et al., 2018).

Irrespective of evidence that HosA indirectly regulates SM production via activation of TFs or histone modifiers and directly via deacetylation of $\mathrm{H} 3 \mathrm{~K} 9$ and $\mathrm{H} 4 \mathrm{~K} 16$, we cannot exclude additional target sites on histones not addressed in our ChIP strategy. One out of further possible targets that was described to be hyper-acetylated under overexpression of the histone acetyltransferase EsaA is $\mathrm{H} 4 \mathrm{~K} 12$, leading to an increased transcription of SM clusters including the otherwise silent OA cluster (Soukup et al., 2012).

Interestingly, expression of silent SMs such as orsellinic acid, lecanoric acid, and the two yellow-orange cathepsin $\mathrm{K}$ inhibitors F-9775A and F9775B was also induced, when A. nidulans was grown in co-culture with the bacterial species S. hygroscopicus (Schroeckh et al., 2009). Streptomyces ssp. are known as natural producers of TSA suggesting that, in co-culture with Aspergillus, inhibition of HosA via bacterial TSA might add to the induction of the orsellinic acid cluster and, as shown here, also contributes to inhibition of penicillin production. Since antibiotics such as penicillin are natural fungal weapons against competing microorganisms, it is conceivable that their inhibition via secretion of HDAC inhibitors might be an appropriate biological answer of Streptomyces in a chemical warfare of microbes for limited resources.

Moreover, expression of orsellinic acid was also found under limitation of nitrogen and phosphorus (Sarkar et al., 2012). Our analysis revealed that alternative starvation conditions such as zinc, carbon, or nitrogen depletion do not affect orsellinic acid cluster genes with two exceptions, copper- and, more striking, iron-starvation. Both conditions significantly triggered orsellinic acid expression and, to a lower extent, that of other SMs in wildtype strains as well (Figure 2C). In addition, iron limitation showed also synergistic effects with hos $A$ depletion, further increasing the regulatory effect of the hos $A$ mutants. Although the specific interplay between HosA and iron remains to be discovered in detail, RNA-Seq data analysis (focused on genes responsible for fungal iron acquisition) revealed that sidD, encoding an NRPS required for the biosynthesis of triacetyfusarinine $\mathrm{C}$ (TAFC), is among the genes that are suppressed in the hosA mutants (Supplementary Figure S3). Since TAFC is essential for the synthesis of extracellular siderophores required for the mobilization of extracellular iron under iron starvation (Schrettl et al., 2008), it is plausible that diminished availability of intracellular iron contributes to the observed transcriptional effects of the SM clusters in hosA delta strains. Indeed, an iron-depending production of SMs was quite recently demonstrated for $p p z A$ deletion strains of $A$. fumigatus. $p p z A$ codes for the catalytic subunit of a protein phosphatase $(\mathrm{PpZ})$ that was discussed as another epigenetic regulator of chromatin structure of the gene clusters affected (Manfiolli et al., 2017). The impact of PpZ on the production of SMs such as fumiquilazoline A, fumagillin, and helvolic acid was as well depending on the disposability of iron. PpzA thereby perturbed the response to iron assimilation of the fungus affecting its siderophore production. Moreover, data of other groups imply that several SMs are subject to iron-dependent regulation by SreA and HapX, two major transcription factors of iron homeostasis, confirming its importance for the production of fungal metabolites (Wiemann et al., 2014).

In addition to the SMs discussed in detail above, our experiments revealed that several other clusters are specifically affected in hos A mutants. Among them the cichorine cluster, responsible for the production of a phytotoxin (Sanchez et al., 2012) and the aspercryptin cluster, responsible for the production of a lipoprotein family. The latter was discovered quite recently by an HDAC inhibitor-based strategy (Chiang et al., 2016). Interestingly, two of these lipoproteins, aspercryptin A1 and B2, were identified and characterized by comparative MS analysis 
and extensive NMR of a wildtype strain versus an RpdA knockdown mutant (Henke et al., 2016). Moderate depletion of this essential class 1 HDAC thereby led to hyper-acetylation of bulk chromatin increasing the expression of these two metabolites. This observation suggests that aspercryptins are silenced by RpdA as well. Unfortunately, however, nothing is known about the impact of RpdA on other HosA-affected (cryptic) SM clusters. One of those clusters is the INP cluster, comprising seven genes (AN3490 to AN3496) on chromosome II. Although silent under laboratory growth conditions, the corresponding metabolite could be characterized by overexpressing its internal regulator $s c p R$ (Bergmann et al., 2010). Interestingly, $s c p R$ overexpression led to a regulatory crosstalk inducing another cluster on chromosome VIII responsible for asperfuranone (AF) biosynthesis. Although our hosA deletion strains showed an early and strong induction of all seven INP cluster genes, the AF cluster remained unaffected at $24 \mathrm{~h}$ of growth. After $60 \mathrm{~h}$, however, the AF cluster genes $a f_{o} E$ and $a f_{o} G$ and their activator $a f_{o} A$ were induced in hos $A$ mutants suggesting, that induction of the AF cluster by $s c p R$ occurs with a time delay.

In $24 \mathrm{~h}$ cultures, another cryptic SM cluster (spanning genes AN2030 to AN2036) was significantly upregulated in hosA mutant strains. Although two of these genes, $p k h A$ (AN2032) and $p k h B$ (AN2035), could be characterized by overexpression as PKS encoding sequences, the final products of this cluster are waiting to be discovered (Ahuja et al., 2012), illustrating an example, how deletion of hosA might contribute to the characterization of still unknown compounds of filamentous fungi.

Very recently, a few other TFs were identified that, when deleted, resulted in induction or repression of SM production. For instance, inactivation of three novel regulators, LaeB, SntB, and HamI, resulted in a complete loss of aflatoxin production in A. flavus (Pfannenstiel et al., 2017), whereas deletion of $m c r A$, a gene encoding for a multicluster regulator present in several fungal species, induced the production of at least 10 small bioactive molecules and allowed the identification of three unknown compounds of $A$. nidulans (Oakley et al., 2016). Nevertheless, data revealed that McrA and a second major regulator of SMs in fungi, LaeA (Bok and Keller, 2004), are affecting fewer than $50 \%$ of the SM gene clusters known, indicating that several other important key players are involved in the complex regulation of fungal SMs. Our data suggest that HosA actually is one of those players.

\section{CONCLUSION}

In conclusion, our results have revealed Hos A a novel master regulator of SMs in A. nidulans that, when deleted or inhibited, represents a promising possibility to open the fungal portfolio rife with bioactive molecules for possible medical applications.

\section{AUTHOR CONTRIBUTIONS}

AP, BF, and IB generated the data. SG, GB, and IB conceived and designed the experiments. IB, AP, and SG analyzed the data. SG, $\mathrm{BF}, \mathrm{AP}$, and IB wrote the manuscript.

\section{FUNDING}

This work was funded by the Austrian Science Foundation (P24803) (SG).

\section{ACKNOWLEDGMENTS}

We are grateful to Gerhard Braus for providing TAP constructs. Hubertus Haas for discussing the results, and Peter Loidl for critical reading of the manuscript.

\section{SUPPLEMENTARY MATERIAL}

The Supplementary Material for this article can be found online at: https://www.frontiersin.org/articles/10.3389/fmicb.2018. 02212/full\#supplementary-material

FIGURE S1 | Sensibility of hosA mutants compared to complemented strains as tested by antifungigrams (ETEST). For sensibility testing against antifungal substances, ETEST strips loaded with an increasing concentration of the antifungals voriconazole, amphotericin, caspofungin, and fluconazole were applied to plates with spores of hosA deletion strains and wt according to the manufacturer's instructions of the test-strips. After incubation for 24 (not shown) and $48 \mathrm{~h}$ at $37^{\circ} \mathrm{C}$, the sensibility was evaluated (see magnification of the fluconazole strip below).

FIGURE S2 | Phenotypical analysis of HosA overexpression strains. His tagged HosA was overexpressed in Aspergillus nidulans strains TBF117 (hosAoe1) and TBF122 (hosAoe2) under control of the heterologous xylanase promoter (xylPp) in submerged culture (GMM) supplemented with $1 \%$ xylose (i) for $48 \mathrm{~h}$ at $37^{\circ} \mathrm{C}$. In order to verify HosA overexpression, crude protein extract was blotted and probed with an anti-HosA antibody. A 55- and 77-kDa marker protein is shown (lane M). A wildtype strain was used to confirm the specificity of the antibody. $x y / P p$ repressing conditions ( $r$, no xylose added) were used as negative control (A). Phenotype of HosA overexpressing strains were subsequently assessed under $x y I P p$ inductive and repressive conditions on agar plates. hosAoe1 and 2, a hosA-complemented (hos $A c$ ), and a hos $A$ deletion ( $\triangle$ hos $A$ ) strain were grown for $48 \mathrm{~h}$ at $37^{\circ} \mathrm{C}$ on $\mathrm{GMM}$ with (i) or without (r) $1 \%$ xylose, before the colonies were compared to a wildtype strain (B).

FIGURE S3 | Heatmap of the expression levels of 70 confirmed or predicted key enzymes (polyketide synthases or non-ribosomal peptide synthetases) of secondary metabolite gene clusters in hos A mutants and wildtype grown at $37^{\circ} \mathrm{C}$. The FPKM + 1 values were log10-transformed and genes were ranked based on their aberration in transcription in mutants versus wildtype of short-term (24 h) or long-term (60 h) cultures, respectively. The blue box indicates enzymes for which differential expression has been confirmed by Northern blot analysis. Blue arrowheads indicate 25 selected genes that were subsequently analyzed together with their adjacent coding sequences in Supplementary Table S7.

FIGURE S4 | Subcellular localization of HosA and RpdA in HosA-GFP and RpdA-GFP expression strains. Strains were grown under $0.1 \%$ xylose to achieve moderate expression of the recombinant HDACs RpdA and HosA, respectively. DNA was stained with DAPI. RpdA was predominantly localized in the nucleus whereas HosA could be detected in both, the nucleus and the cytoplasm.

TABLE S1 | Genotypes of fungal and bacterial strains used in this study. The lab name and the pseudonym of fungal strains used in this manuscript are specified. Genetic characteristic is indicated as follows: hosA::argB indicates the replacement of hos $A$ by the auxotrophic marker gene encoding ornithine transcarbamylase $(\arg B)$ used for selection of transformants, xylpP:hosA:His:argB; argB2 refers to a copy of His-tagged hosA under control of the heterologous xylanase promoter ( $x y / P \mathrm{p})$ randomly integrated into the genome in addition to endogenous hos $A$. For the generation of some mutants, a phleomycin resistance gene $(b / e R)$ was used instead of $\arg B$ as selection marker. Strains $\mathrm{H} 1$ and 
TBF53.1 or TBF53.2 were used for sexual crosses to gain hdaA/hosA double knock out mutants, $x$ is indicating sexual crosses of the corresponding strains.

TABLE S2 | Oligonucleotides used for qPCR and generation of probes. Primers for qPCR of the ChIP analysis are listed; three different regions (T1, T2, T3) in each of the corresponding SM cluster genes were used for quantification of precipitated DNA (left column). Primer pairs used to generate Dig-dUTP-labeled DNA probes for transcriptional analysis in Northern blots are shown (right column). PN, penicillin; OA, orsellinic acid; CC, cichorine; AC, aspercryptin.

TABLE S3 | TopHat summary of mapped reads of each RNA-Seq sample. Table shows the amount of input reads (reached from Illumina HiSeq Single Read sequencing), successfully mapped reads and number of reads aligned multiple times to the reference genome.

TABLE S4 | Genes represented in Venn-diagrams and used for FunCat classification ontology analysis. Listed genes are the result of filtering Cuffdiff results of 10.820 genes for Cuffdiff-significance, FDR $(<0.05)$ and $\log 2 F C(\geq 2)$. Genes that are regarded as being differentially expressed between wildtype and hos $A$ deletion mutant grown for 24 and $60 \mathrm{~h}$ are shown. Genes are divided into up- and down-regulated transcripts including those that are overlapping in both conditions.

TABLE S5 | Collection of 70 confirmed or predicted genes encoding polyketide synthases or non-ribosomal peptide synthetases responsible for the biosynthesis of secondary metabolites. Detailed information about the type of enzyme, gene description, locus of the coding sequence in the genome and the compound, which is biosynthesized by the gene cluster is given (data sheet 1). Expression

\section{REFERENCES}

Abshiru, N., Rajan, R. E., Verreault, A., and Thibault, P. (2016). Unraveling sitespecific and combinatorial histone modifications using high-resolution mass spectrometry in histone deacetylase mutants of fission yeast. J. Proteome Res. 15, 2132-2142. doi: 10.1021/acs.jproteome.5b01156

Ahuja, M., Chiang, Y.-M., Chang, S.-L., Praseuth, M. B., Entwistle, R., Sanchez, J. F., et al. (2012). Illuminating the diversity of aromatic polyketide synthases in Aspergillus nidulans. J. Am. Chem. Soc. 134, 8212-8221. doi: 10.1021/ja3016395

Andersen, M. R., Nielsen, J. B., Klitgaard, A., Petersen, L. M., Zachariasen, M., Hansen, T. J., et al. (2013). Accurate prediction of secondary metabolite gene clusters in filamentous fungi. Proc. Natl. Acad. Sci. U.S.A. 110, E99-E107. doi: 10.1073/pnas. 1205532110

Austin, B., Hall, R. M., and Tyler, B. M. (1990). Optimized vectors and selection for transformation of Neurospora crassa and Aspergillus nidulans to bleomycin and phleomycin resistance. Gene 93, 157-162. doi: 10.1016/0378-1119(90) 90152-H

Baidyaroy, D., Brosch, G., Ahn, J. H., Graessle, S., Wegener, S., Tonukari, N. J., et al. (2001). A gene related to yeast HOS2 histone deacetylase affects extracellular depolymerase expression and virulence in a plant pathogenic fungus. Plant Cell 13, 1609-1624. doi: 10.1105/tpc.13.7.1609

Barrett, T., Wilhite, S. E., Ledoux, P., Evangelista, C., Kim, I. F., Tomashevsky, M., et al. (2013). NCBI GEO: archive for functional genomics data sets-update. Nucleic Acids Res. 41, D991-D995. doi: 10.1093/nar/gks1193

Bauer, I., Varadarajan, D., Pidroni, A., Gross, S., Vergeiner, S., Faber, B., et al. (2016). A class 1 histone deacetylase with potential as an antifungal target. mBio 7:e00831-816. doi: 10.1128/mBio.00831-816

Bayram, Ö, Bayram, ÖS., Valerius, O., Jöhnk, B., and Braus, G. H. (2012). Identification of protein complexes from filamentous fungi with tandem affinity purification. Methods Mol. Biol. 944, 191-205. doi: 10.1007/978-1-62703-1226-14

Bergmann, S., Funk, A. N., Scherlach, K., Schroeckh, V., Shelest, E., Horn, U., et al. (2010). Activation of a silent fungal polyketide biosynthesis pathway through regulatory cross talk with a cryptic nonribosomal peptide synthetase gene cluster. Appl. Environ. Microbiol. 76, 8143-8149. doi: 10.1128/AEM.00683-610

Bergmann, S., Schümann, J., Scherlach, K., Lange, C., Brakhage, A. A., and Hertweck, C. (2007). Genomics-driven discovery of PKS-NRPS hybrid metabolites from Aspergillus nidulans. Nat. Chem. Biol. 3, 213-217. doi: 10. 1038/nchembio869 levels in wildtype and hos A mutants after 24 and $60 \mathrm{~h}$ of growth are compared (data sheet 2). Expression confirmed by Northern blot experiments are highlighted in gray.

TABLE S6 | Selected Cuffdiff results of the orsellinic acid, aspercryptin, cichorine and penicillin gene clusters of hosA mutants grown for 24 and $60 \mathrm{~h}$ compared to wildtype. Secondary metabolite gene clusters (gray) flanked by five up- and five downstream genes are shown to confirm the specific regulation of the SM clusters. Sample 1 is compared to sample 2 including the corresponding FPKM value for both samples, the resulting log2fc, the fold change and the $p$-value.

TABLE S7 | Confirmed or putative secondary metabolite gene clusters and adjacent genes. (Predicted) key enzymes such as polyketide synthases or non-ribosomal peptide synthetases of the respective SM clusters are highlighted in the data sheets in gray. Expression levels of the key synthases and at least 20 adjacent genes flanking both sides of each key enzymes were compared between wildtype and hosA mutants of short-term (24 h) and long-term (60 h) cultures. Relating to the log2FC, a cutoff of \pm 1.0 was applied considering the significance criteria (sig) of Cuffdiff.

TABLE S8 | Results of FungiFun2 analysis performed with the FunCat classification ontology option. Genes listed in Supplementary Table S4 were used as input IDs. For each comparison between wildtype and hos A mutant strains grown for 24 and $60 \mathrm{~h}$, genes were divided into up- and downregulated categories. Category and gene-ID summary containing gene-ID, protein name, FunCat ID with description, and $p$-values are shown.

Bertrand, S., Schumpp, O., Bohni, N., Bujard, A., Azzollini, A., Monod, M., et al. (2013). Detection of metabolite induction in fungal co-cultures on solid media by high-throughput differential ultra-high pressure liquid chromatographytime-of-flight mass spectrometry fingerprinting. J. Chromatogr. A 1292, 219-228. doi: 10.1016/j.chroma.2013.01.098

Boedi, S., Reyes-Dominguez, Y., and Strauss, J. (2012). Chromatin immunoprecipitation analysis in filamentous fungi. Methods Mol. Biol. 944, 221-236. doi: 10.1007/978-1-62703-122-6-16

Bok, J. W., Hoffmeister, D., Maggio-Hall, L. A., Murillo, R., Glasner, J. D., and Keller, N. P. (2006). Genomic mining for Aspergillus natural products. Chem. Biol. 13, 31-37. doi: 10.1016/j.chembiol.2005.10.008

Bok, J. W., and Keller, N. P. (2004). LaeA, a regulator of secondary metabolism in Aspergillus spp. Eukaryot. Cell 3, 527-535. doi: 10.1128/EC.3.2.527-535.2004

Brakhage, A. A. (2013). Regulation of fungal secondary metabolism. Nat. Rev. Microbiol. 11, 21-32. doi: 10.1038/nrmicro2916

Brosch, G., Loidl, P., and Graessle, S. (2008). Histone modifications and chromatin dynamics: a focus on filamentous fungi. FEMS Microbiol. Rev. 32, 409-439. doi: 10.1111/j.1574-6976.2007.00100.x

Brown, D. W., Yu, J. H., Kelkar, H. S., Fernandes, M., Nesbitt, T. C., Keller, N. P., et al. (1996). Twenty-five coregulated transcripts define a sterigmatocystin gene cluster in Aspergillus nidulans. Proc. Natl. Acad. Sci. U.S.A. 93, 1418-1422. doi: 10.1073/pnas.93.4.1418

Cai, Q., Tong, S.-M., Shao, W., Ying, S.-H., and Feng, M.-G. (2018). Pleiotropic effects of the histone deacetylase Hos2 linked to H4-K16 deacetylation, H3K56 acetylation, and H2A-S129 phosphorylation in Beauveria bassiana. Cell. Microbiol. 13:e12839. doi: 10.1111/cmi.12839

Chen, H. (2018). VennDiagram: Generate High-Resolution Venn and Euler Plots. Available at: https://CRAN.R-project.org/package=VennDiagram

Chen, X.-F., Kuryan, B., Kitada, T., Tran, N., Li, J.-Y., Kurdistani, S., et al. (2012). The Rpd3 core complex is a chromatin stabilization module. Curr. Biol. 22, 56-63. doi: 10.1016/j.cub.2011.11.042

Chiang, Y.-M., Ahuja, M., Oakley, C. E., Entwistle, R., Asokan, A., Zutz, C., et al. (2016). Development of genetic dereplication strains in Aspergillus nidulans results in the discovery of aspercryptin. Angew. Chem. Int. Ed. Engl. 55, 1662-1665. doi: 10.1002/anie.201507097

Chiang, Y.-M., Szewczyk, E., Davidson, A. D., Keller, N., Oakley, B. R., and Wang, C. C. C. (2009). A gene cluster containing two fungal polyketide synthases encodes the biosynthetic pathway for a polyketide, asperfuranone, in Aspergillus nidulans. J. Am. Chem. Soc. 131, 2965-2970. doi: 10.1021/ja8088185 
Chiang, Y.-M., Szewczyk, E., Nayak, T., Davidson, A. D., Sanchez, J. F., Lo, H.-C., et al. (2008). Molecular genetic mining of the Aspergillus secondary metabolome: discovery of the emericellamide biosynthetic pathway. Chem. Biol. 15, 527-532. doi: 10.1016/j.chembiol.2008.05.010

Chooi, Y.-H., Fang, J., Liu, H., Filler, S. G., Wang, P., and Tang, Y. (2013). Genome mining of a prenylated and immunosuppressive polyketide from pathogenic fungi. Org. Lett. 15, 780-783. doi: 10.1021/ol303435y

Cove, D. J. (1966). The induction and repression of nitrate reductase in the fungus Aspergillus nidulans. Biochim. Biophys. Acta 113, 51-56. doi: 10.1016/S09266593(66)80120-0

Dokmanovic, M., Clarke, C., and Marks, P. A. (2007). Histone deacetylase inhibitors: overview and perspectives. Mol. Cancer Res. 5, 981-989. doi: 10. 1158/1541-7786.MCR-07-0324

Elaut, G., Rogiers, V., and Vanhaecke, T. (2007). The pharmaceutical potential of histone deacetylase inhibitors. Curr. Pharm. Des. 13, 2584-2620. doi: 10.2174/ 138161207781663064

El-Hawary, S., Sayed, A., Mohammed, R., Hassan, H., Zaki, M., Rateb, M., et al. (2018). Epigenetic modifiers induce bioactive phenolic metabolites in the marine-derived fungus Penicillium brevicompactum. Mar. Drugs 16:E253. doi: $10.3390 / \mathrm{md} 16080253$

Fan, A., Mi, W., Liu, Z., Zeng, G., Zhang, P., Hu, Y., et al. (2017). Deletion of a histone acetyltransferase leads to the pleiotropic activation of natural products in Metarhizium robertsii. Org. Lett. 19, 1686-1689. doi: 10.1021/acs.orglett. $7 \mathrm{~b} 00476$

Fox, E. M., and Howlett, B. J. (2008). Secondary metabolism: regulation and role in fungal biology. Curr. Opin. Microbiol. 11, 481-487. doi: 10.1016/j.mib.2008. 10.007

Gacek, A., and Strauss, J. (2012). The chromatin code of fungal secondary metabolite gene clusters. Appl. Microbiol. Biotechnol. 95, 1389-1404. doi: 10. 1007/s00253-012-4208-4208

Glozak, M. A., Sengupta, N., Zhang, X., and Seto, E. (2005). Acetylation and deacetylation of non-histone proteins. Gene 363, 15-23. doi: 10.1016/j.gene. 2005.09.010

Graessle, S., Dangl, M., Haas, H., Mair, K., Trojer, P., Brandtner, E. M., et al. (2000). Characterization of two putative histone deacetylase genes from Aspergillus nidulans. Biochim. Biophys. Acta 1492, 120-126. doi: 10.1016/S0167-4781(00) 00093-2

Harvey, A. L., Edrada-Ebel, R., and Quinn, R. J. (2015). The re-emergence of natural products for drug discovery in the genomics era. Nat. Rev. Drug Discov. 14, 111-129. doi: 10.1038/nrd4510

Hautbergue, T., Jamin, E. L., Debrauwer, L., Puel, O., and Oswald, I. P. (2018). From genomics to metabolomics, moving toward an integrated strategy for the discovery of fungal secondary metabolites. Nat. Prod. Rep. 35, 147-173. doi: $10.1039 / \mathrm{c} 7 \mathrm{np} 00032 \mathrm{~d}$

Henke, M. T., Soukup, A. A., Goering, A. W., McClure, R. A., Thomson, R. J., Keller, N. P., et al. (2016). New aspercryptins, lipopeptide natural products, revealed by HDAC inhibition in Aspergillus nidulans. ACS Chem. Biol. 11, 2117-2123. doi: 10.1021/acschembio.6b00398

Inglis, D. O., Binkley, J., Skrzypek, M. S., Arnaud, M. B., Cerqueira, G. C., Shah, P., et al. (2013). Comprehensive annotation of secondary metabolite biosynthetic genes and gene clusters of Aspergillus nidulans, A. fumigatus, A. niger and A. oryzae. BMC Microbiol. 13:91. doi: 10.1186/1471-2180-13-91

Karmodiya, K., Krebs, A. R., Oulad-Abdelghani, M., Kimura, H., and Tora, L. (2012). H3K9 and H3K14 acetylation co-occur at many gene regulatory elements, while $\mathrm{H} 3 \mathrm{~K} 14 \mathrm{ac}$ marks a subset of inactive inducible promoters in mouse embryonic stem cells. BMC Genomics 13:424. doi: 10.1186/1471-216413-424

Khaldi, N., Seifuddin, F. T., Turner, G., Haft, D., Nierman, W. C., Wolfe, K. H., et al. (2010). SMURF: genomic mapping of fungal secondary metabolite clusters. Fungal Genet. Biol. 47, 736-741. doi: 10.1016/j.fgb.2010.06.003

Klitgaard, A., Nielsen, J. B., Frandsen, R. J. N., Andersen, M. R., and Nielsen, K. F. (2015). Combining stable isotope labeling and molecular networking for biosynthetic pathway characterization. Anal. Chem. 87, 6520-6526. doi: 10.1021/acs.analchem.5b01934

Kolde, R. (2015). pheatmap: Pretty Heatmaps. Available at: https:/CRAN.Rproject.org/package $=$ pheatmap

Kong, X., van Diepeningen, A. D., van der Lee, T. A. J., Waalwijk, C., Xu, J., $\mathrm{Xu}$, J., et al. (2018). The Fusarium graminearum histone acetyltransferases are important for morphogenesis, DON biosynthesis, and pathogenicity. Front. Microbiol. 9:353. doi: 10.3389/fmicb.2018.00654

Kurdistani, S. K., and Grunstein, M. (2003). Histone acetylation and deacetylation in yeast. Nat. Rev. Mol. Cell Biol. 4, 276-284. doi: 10.1038/nrm1075

Lamoth, F., Juvvadi, P. R., and Steinbach, W. J. (2015). Histone deacetylase inhibition as an alternative strategy against invasive aspergillosis. Front. Microbiol. 6:96. doi: 10.3389/fmicb.2015.00096

Lee, I., Oh, J.-H., Shwab, E. K., Dagenais, T. R. T., Andes, D., and Keller, N. P. (2009). HdaA, a class 2 histone deacetylase of Aspergillus fumigatus, affects germination and secondary metabolite production. Fungal Genet. Biol. 46, 782-790. doi: 10.1016/j.fgb.2009.06.007

Leipe, D. D., and Landsman, D. (1997). Histone deacetylases, acetoin utilization proteins and acetylpolyamine amidohydrolases are members of an ancient protein superfamily. Nucleic Acids Res. 25, 3693-3697. doi: 10.1093/nar/25.18. 3693

Lin, H., Lyu, H., Zhou, S., Yu, J., Keller, N. P., Chen, L., et al. (2018). Deletion of a global regulator LaeB leads to the discovery of novel polyketides in Aspergillus nidulans. Org. Biomol. Chem. 16, 4973-4976. doi: 10.1039/c8ob01326h

Liu, I.-C., Chiu, S.-W., Lee, H.-Y., and Leu, J.-Y. (2012). The histone deacetylase Hos2 forms an Hsp42-dependent cytoplasmic granule in quiescent yeast cells. Mol. Biol. Cell 23, 1231-1242. doi: 10.1091/mbc.E11-09-0752

Liu, W., Xie, Y., Ma, J., Luo, X., Nie, P., Zuo, Z., et al. (2015). IBS: an illustrator for the presentation and visualization of biological sequences. Bioinformatics 31, 3359-3361. doi: 10.1093/bioinformatics/btv362

Loidl, P. (1994). Histone acetylation: facts and questions. Chromosoma 103, 441-449. doi: 10.1007/BF00337382

Lubertozzi, D., and Keasling, J. D. (2006). Marker and promoter effects on heterologous expression in Aspergillus nidulans. Appl. Microbiol. Biotechnol. 72, 1014-1023. doi: 10.1007/s00253-006-0368-368

Manfiolli, A. O., de Castro, P. A., dos Reis, T. F., Dolan, S., Doyle, S., Jones, G., et al. (2017). Aspergillus fumigatus protein phosphatase PpzA is involved in iron assimilation, secondary metabolite production, and virulence. Cell. Microbiol. 19:e12770. doi: 10.1111/cmi.12770

Martínez-Balbás, M. A., Bauer, U. M., Nielsen, S. J., Brehm, A., and Kouzarides, T. (2000). Regulation of E2F1 activity by acetylation. EMBO J. 19, 662-671. doi: 10.1093/emboj/19.4.662

Netzker, T., Fischer, J., Weber, J., Mattern, D. J., König, C. C., Valiante, V., et al. (2015). Microbial communication leading to the activation of silent fungal secondary metabolite gene clusters. Front. Microbiol. 6:299. doi: 10.3389/fmicb. 2015.00299

Newman, D. J., and Cragg, G. M. (2012). Natural products as sources of new drugs over the 30 years from 1981 to 2010. J. Nat. Prod. 75, 311-335. doi: $10.1021 / \mathrm{np} 200906 \mathrm{~s}$

Nielsen, M. L., Nielsen, J. B., Rank, C., Klejnstrup, M. L., Holm, D. K., Brogaard, K. H., et al. (2011). A genome-wide polyketide synthase deletion library uncovers novel genetic links to polyketides and meroterpenoids in Aspergillus nidulans. FEMS Microbiol. Lett. 321, 157-166. doi: 10.1111/j.1574-6968.2011. 02327.x

Nützmann, H.-W., Reyes-Dominguez, Y., Scherlach, K., Schroeckh, V., Horn, F., Gacek, A., et al. (2011). Bacteria-induced natural product formation in the fungus Aspergillus nidulans requires Saga/Ada-mediated histone acetylation. Proc. Natl. Acad. Sci. U.S.A. 108, 14282-14287. doi: 10.1073/pnas.1103523108

Oakley, C. E., Ahuja, M., Sun, W.-W., Entwistle, R., Akashi, T., Yaegashi, J., et al. (2016). Discovery of McrA, a master regulator of Aspergillus secondary metabolism. Mol. Microbiol. 103, 347-365. doi: 10.1111/mmi.13562

Palmer, J. M., and Keller, N. P. (2010). Secondary metabolism in fungi: does chromosomal location matter? Curr. Opin. Microbiol. 13, 431-436. doi: 10.1016/ j.mib.2010.04.008

Pfaller, M. A., Messer, S. A., Georgopapadakou, N., Martell, L. A., Besterman, J. M., and Diekema, D. J. (2009). Activity of MGCD290, a Hos2 histone deacetylase inhibitor, in combination with azole antifungals against opportunistic fungal pathogens. J. Clin. Microbiol. 47, 3797-3804. doi: 10.1128/JCM.00618-619

Pfannenstiel, B. T., Zhao, X., Wortman, J., Wiemann, P., Throckmorton, K., Spraker, J. E., et al. (2017). Revitalization of a forward genetic screen identifies three new regulators of fungal secondary metabolism in the genus Aspergillus. mBio 8:e01246-1217. doi: 10.1128/mBio.01246-1217

Pitt, J. I. (2000). Toxigenic fungi and mycotoxins. Br. Med. Bull. 56, 184-192. doi: $10.1258 / 0007142001902888$ 
Priebe, S., Kreisel, C., Horn, F., Guthke, R., and Linde, J. (2015). FungiFun2: a comprehensive online resource for systematic analysis of gene lists from fungal species. Bioinformatics 31, 445-446. doi: 10.1093/bioinformatics/btu627

Ruepp, A., Zollner, A., Maier, D., Albermann, K., Hani, J., Mokrejs, M., et al. (2004). The FunCat, a functional annotation scheme for systematic classification of proteins from whole genomes. Nucleic Acids Res. 32, 5539-5545. doi: 10.1093/ nar/gkh894

Sanchez, J. F., Entwistle, R., Corcoran, D., Oakley, B. R., and Wang, C. C. C. (2012). Identification and molecular genetic analysis of the cichorine gene cluster in Aspergillus nidulans. Medchemcomm 3, 997-1002. doi: 10.1039/C2MD20055D

Sarkar, A., Funk, A. N., Scherlach, K., Horn, F., Schroeckh, V., Chankhamjon, P., et al. (2012). Differential expression of silent polyketide biosynthesis gene clusters in chemostat cultures of Aspergillus nidulans. J. Biotechnol. 160, 64-71. doi: 10.1016/j.jbiotec.2012.01.015

Schrettl, M., Kim, H. S., Eisendle, M., Kragl, C., Nierman, W. C., Heinekamp, T., et al. (2008). SreA-mediated iron regulation in Aspergillus fumigatus. Mol. Microbiol. 70, 27-43. doi: 10.1111/j.1365-2958.2008.06376.x

Schroeckh, V., Scherlach, K., Nützmann, H.-W., Shelest, E., Schmidt-Heck, W., Schuemann, J., et al. (2009). Intimate bacterial-fungal interaction triggers biosynthesis of archetypal polyketides in Aspergillus nidulans. Proc. Natl. Acad. Sci. U.S.A. 106, 14558-14563. doi: 10.1073/pnas.0901870106

Shwab, E. K., Bok, J. W., Tribus, M., Galehr, J., Graessle, S., and Keller, N. P. (2007). Histone deacetylase activity regulates chemical diversity in Aspergillus. Eukaryot. Cell 6, 1656-1664. doi: 10.1128/EC.00186-187

Smith, W. L., and Edlind, T. D. (2002). Histone deacetylase inhibitors enhance Candida albicans sensitivity to azoles and related antifungals: correlation with reduction in CDR and ERG upregulation. Antimicrob. Agents Chemother. 46, 3532-3539. doi: 10.1128/AAC.46.11.3532-3539.2002

Soukup, A. A., Chiang, Y.-M., Bok, J. W., Reyes-Dominguez, Y., Oakley, B. R., Wang, C. C. C., et al. (2012). Over expression of the Aspergillus nidulans histone 4 acetyltransferase EsaA increases activation of secondary metabolite production. Mol. Microbiol. 86, 314-330. doi: 10.1111/j.1365-2958.2012. 08195.x

Strahl, B. D., and Allis, C. D. (2000). The language of covalent histone modifications. Nature 403, 41-45. doi: 10.1038/47412

Studt, L., Rösler, S. M., Burkhardt, I., Arndt, B., Freitag, M., Humpf, H.-U., et al. (2016). Knock-down of the methyltransferase Kmt6 relieves H3K27me3 and results in induction of cryptic and otherwise silent secondary metabolite gene clusters in Fusarium fujikuroi. Environ. Microbiol. 18, 4037-4054. doi: 10.1111/ 1462-2920.13427

Studt, L., Schmidt, F. J., Jahn, L., Sieber, C. M. K., Connolly, L. R., Niehaus, E.M., et al. (2013). Two histone deacetylases, FfHda1 and FfHda2, are important for Fusarium fujikuroi secondary metabolism and virulence. Appl. Environ. Microbiol. 79, 7719-7734. doi: 10.1128/AEM.01557-1513

Todd, R. B., Davis, M. A., and Hynes, M. J. (2007). Genetic manipulation of Aspergillus nidulans: meiotic progeny for genetic analysis and strain construction. Nat. Protoc. 2, 811-821. doi: 10.1038/nprot.2007.112

Trapnell, C., Roberts, A., Goff, L., Pertea, G., Kim, D., Kelley, D. R., et al. (2012). Differential gene and transcript expression analysis of RNA-seq experiments with TopHat and Cufflinks. Nat. Protoc. 7, 562-578. doi: 10.1038/nprot.20 12.016

Tribus, M., Bauer, I., Galehr, J., Rieser, G., Trojer, P., Brosch, G., et al. (2010). A novel motif in fungal class 1 histone deacetylases is essential for growth and development of Aspergillus. Mol. Biol. Cell 21, 345-353. doi: 10.1091/mbc.E0908-0750

Tribus, M., Galehr, J., Trojer, P., Brosch, G., Loidl, P., Marx, F., et al. (2005). HdaA, a major class 2 histone deacetylase of Aspergillus nidulans, affects growth under conditions of oxidative stress. Eukaryot. Cell 4, 1736-1745. doi: 10.1128/EC.4. 10.1736-1745.2005

Trojer, P., Brandtner, E. M., Brosch, G., Loidl, P., Galehr, J., Linzmaier, R., et al. (2003). Histone deacetylases in fungi: novel members, new facts. Nucleic Acids Res. 31, 3971-3981. doi: 10.1093/nar/gkg473

van der Lee, T. A. J., and Medema, M. H. (2016). Computational strategies for genome-based natural product discovery and engineering in fungi. Fungal Genet. Biol. 89, 29-36. doi: 10.1016/j.fgb.2016.01.006

Vansteelandt, M., Blanchet, E., Egorov, M., Petit, F., Toupet, L., Bondon, A., et al. (2013). Ligerin, an antiproliferative chlorinated sesquiterpenoid from a marine-derived Penicillium strain. J. Nat. Prod. 76, 297-301. doi: 10.1021/np30 07364

Verdin, E., and Ott, M. (2015). 50 years of protein acetylation: from gene regulation to epigenetics, metabolism and beyond. Nat. Rev. Mol. Cell Biol. 16, 258-264. doi: $10.1038 / \mathrm{nrm} 3931$

Wang, A., Kurdistani, S. K., and Grunstein, M. (2002). Requirement of Hos2 histone deacetylase for gene activity in yeast. Science 298, 1412-1414. doi: $10.1126 /$ science. 1077790

Wickham, H. (2009). ggplot2: Elegant Graphics for Data Analysis. New York, NY: Springer-Verlag. doi: 10.1007/978-0-387-98141-3

Wickham, H., Francois, R., Henry, L., and Müller, K. (2017). dplyr: A Grammar of Data Manipulation. Available at: https://CRAN.R-project.org/package=dplyr

Wiemann, P., Lechner, B. E., Baccile, J. A., Velk, T. A., Yin, W.-B., Bok, J. W., et al. (2014). Perturbations in small molecule synthesis uncovers an iron-responsive secondary metabolite network in Aspergillus fumigatus. Front. Microbiol. 5:530. doi: $10.3389 /$ fmicb. 2014.00530

Wirén, M., Silverstein, R. A., Sinha, I., Walfridsson, J., Lee, H.-M., Laurenson, P., et al. (2005). Genome wide analysis of nucleosome density histone acetylation and HDAC function in fission yeast. EMBO J. 24, 2906-2918. doi: 10.1038/sj. emboj.7600758

Yaegashi, J., Oakley, B. R., and Wang, C. C. C. (2014). Recent advances in genome mining of secondary metabolite biosynthetic gene clusters and the development of heterologous expression systems in Aspergillus nidulans. J. Ind. Microbiol. Biotechnol. 41, 433-442. doi: 10.1007/s10295-013-1386-Z

Yeh, H.-H., Chiang, Y.-M., Entwistle, R., Ahuja, M., Lee, K.-H., Bruno, K. S., et al. (2012). Molecular genetic analysis reveals that a nonribosomal peptide synthetase-like (NRPS-like) gene in Aspergillus nidulans is responsible for microperfuranone biosynthesis. Appl. Microbiol. Biotechnol. 96, 739-748. doi: 10.1007/s00253-012-4098-4099

Yoon, S., and Eom, G. H. (2016). HDAC and HDAC inhibitor: from cancer to cardiovascular diseases. Chonnam Med. J. 52, 1-11. doi: 10.4068/cmj.2016. 52.1 .1

Zhang, L., Zhang, J., Jiang, Q., Zhang, L., and Song, W. (2018). Zinc binding groups for histone deacetylase inhibitors. J. Enzyme Inhib. Med. Chem. 33, 714-721. doi: 10.1080/14756366.2017.1417274

Conflict of Interest Statement: The authors declare that the research was conducted in the absence of any commercial or financial relationships that could be construed as a potential conflict of interest.

Copyright (c) 2018 Pidroni, Faber, Brosch, Bauer and Graessle. This is an open-access article distributed under the terms of the Creative Commons Attribution License (CC BY). The use, distribution or reproduction in other forums is permitted, provided the original author(s) and the copyright owner(s) are credited and that the original publication in this journal is cited, in accordance with accepted academic practice. No use, distribution or reproduction is permitted which does not comply with these terms. 